\title{
Identification of HIV-Reservoir Cells with Reduced Susceptibility to
}

\section{Antibody-Dependent Immune Response}

\author{
Antonio Astorga-Gamaza ${ }^{1}$, Judith Grau-Expósito ${ }^{1}$, Joaquín Burgos ${ }^{1}$, Jordi Navarro ${ }^{1}$, Adrià Curran ${ }^{1}$, \\ Bibiana Planas ${ }^{1}$, Paula Suanzes ${ }^{1}$, Vicenç Falcó ${ }^{1}$, Meritxell Genescà ${ }^{1}$, and Maria J. Buzon ${ }^{1 *}$ \\ ${ }^{1}$ Infectious Disease Department, Hospital Universitari Vall d'Hebron, Institut de Recerca (VHIR), \\ Universitat Autònoma de Barcelona, Barcelona, Spain.
}

Running title: Immune resistance of HIV-infected cells to NK-ADCC killing

Corresponding authors*: Maria J. Buzon (mariajose.buzon@vhir.org)

\section{Abstract}

HIV establishes a persistent infection in heterogeneous cell reservoirs, which can be maintained by different mechanisms including cellular proliferation, and represent the main obstacle to curing the infection. The expression of the Fcy receptor CD32 has been identified as a marker of the active cell reservoirs in people on antiretroviral therapy, but if its expression has any role in conferring advantage for viral persistence is unknown. Here, we report that HIV-infected cells expressing CD32 have reduced susceptibility to natural killer (NK) antibody-dependent cell cytotoxicity (ADCC) by a mechanism compatible with the suboptimal binding of HIV-specific antibodies. Infected CD32 cells have increased proliferative capacity in the presence of immune complexes, and are more resistant to strategies directed to potentiate NK function. Remarkably, reactivation of the latent reservoir from antiretroviral-treated people living with HIV increases the pool of infected CD32 cells, which are largely resistant to the ADCC immune mechanism. Thus, we report the existence of reservoir cells that evade part of the NK immune response through the expression of CD32. 
Human immunodeficiency virus (HIV) establishes a persistent infection for which, nowadays, there is no available cure. Despite huge advances on the optimization of antiretroviral therapy (ART), which leads to suppression of viral replication, ART does not fully eliminate the virus from the human body nor can completely solve the persistent inflammation caused by HIV [1]. Importantly, ART discontinuation leads to viral rebound from diverse anatomical sites and cell subsets containing replication-competent virus, representing the main obstacle in achieving cure [2].

The HIV reservoir has a complex and heterogeneous nature, where each of the subsets that compose the viral reservoir contributes differently to viral persistence; i.e. central memory cells are the main population contributing to the total reservoir size [3], effector memory cells support HIV transcription [4], and memory stem cells and resident memory $T$ cells are potentially long-lived niches for HIV $[5,6]$. Unfortunately, knowledge on the establishment, maintenance, and composition of the reservoir remains incomplete, and the identification of markers to exclusively target persistent HIV-infected cells remains elusive $[7,8]$. In this regard, the molecule CD32, a low-

41 affinity receptor for the constant fraction of immunoglobulin $\mathrm{G}(\mathrm{Fc} \gamma \mathrm{R}-\mathrm{Ila})$, was proposed as a marker

42 of HIV reservoir cells [9]. While those results were questioned later by the identification of 43 experimental artifacts [10,11], several new studies partially corroborated the original findings; 44 higher levels of viral DNA within the $T_{C D 32}$ population were reported after applying a very stringent 45 cell isolation protocol [12] and CD32 was identified as a marker of transcriptionally active persistent 
HIV-infected cells, both in blood and in the main reservoir tissues, namely the lymph nodes and the gastrointestinal tract $[6,13-16]$. Importantly, whether or not CD32 is a marker of latent or transcriptionally active infection, infected $\mathrm{CD}^{+} \mathrm{T}$ cells expressing $\mathrm{CD} 32$ contain replicationcompetent HIV and are found in long-term ART-treated people living with HIV (PLWH) [6, 9, 12-15, $17,18]$. The cell markers CD20 [19] and CD30 [20] have been shown to also identify transcriptionally active HIV cells in samples from ART-suppressed PLWH. Whether these transcriptionally active HIVinfected cells persist in the body and are not targeted by host immune responses remains unknown.

Natural killer (NK) cells are lymphocytes that can eliminate cancer cells or virally-infected cells without prior antigen sensitization. They constitute an important arm of the immune system, not only by a direct cytotoxic effect on aberrant cells but also by the modulation of the adaptive immune responses. NK cells kill target cells by several mechanisms, such as natural cytotoxicity (NC), recognizing stress ligands expressed on the surface of infected cells, or by antibody-dependent cell cytotoxicity (ADCC), driven by antibodies that bind to target cells [21]. The decision of NK cells to kill or not to kill a target cell depends on the balance between activating and inhibitory signals received from the interaction with the target cell [22]. Among relevant NK receptors, we find natural cytotoxicity receptors such as NKp46, NKp30, NKp44, different Killer-cell immunoglobulin-like (KIRs), and lectin-like receptors such as NKG2A or NKG2C. Other important activating receptors for NK activity are NKG2D and DNAM-1, whose known ligands are the major histocompatibility complex (MHC) class I-related molecules MICA/B and the UL16-binding proteins or CD155 and CD112, respectively. Further, expression of the inhibitory receptor NKG2A is known to impact NK effector responses through its interaction with HLA-E molecules [23]. In this sense, therapeutic interventions blocking this interaction represent promising tools to potentiate NK cell immune responses during different pathologies [24, 25]. Importantly, HIV infection causes NK cell dysfunction, which is not completely restored by ART $[26,27]$. NK cells play an important role in containing viral replication during early infection and shaping adaptive immune responses during chronic infection [28]. However, the role of NK cells in controlling the viral reservoir in PLWH on ART remains undefined. Mounting evidence supports the importance of NK cell function in shaping the HIV reservoir size, for example through the induction of interferon (IFN- $\gamma$ ) and the expression of certain activating receptors [29]. Moreover, proportions of NK cells inversely correlated with HIV-1 DNA reservoir levels in a clinical study designed to disrupt viral latency [30]. Importantly, during chronic infection, several strategies used by HIV to evade NK cell immune responses have been described, including the modulation of HLA class I molecules expression at the surface of infected cells [31]. However, 
immune evasion mechanisms of HIV-infected reservoir cells to NK cell-mediated killing have not been identified.

Here we show that CD32 expression on HIV-infected cells confers a reduced susceptibility to NK cell-mediated ADCC killing by a mechanism compatible with a reduced binding of HIV-specific antibodies required for this mechanism. Importantly, this immune-resistant mechanism is also observed in latently HIV-infected cells from ART-treated PLWH after viral reactivation, providing a plausible explanation for the maintenance of transcriptionally active HIV-infected cells in ARTtreated PLWH.

\section{RESULTS}

\section{Susceptibility of HIV-reservoir cell subsets to NK immune response}

While it is clear that ADCC, largely mediated by NK cells, is an important protective mechanism against HIV and SIV infection [32-34], the capacity of this immune mechanism to limit the infection of different T cell subsets composing the viral reservoir is currently unknown. Thus, we first assessed the intrinsic susceptibility of Naïve $\left(T_{N A}\right)$, Stem Cell Memory $\left(T_{S C M}\right)$, Central Memory $\left(T_{C M}\right)$, Effector Memory $\left(T_{E M}\right), T_{C D 20}{ }^{\operatorname{dim}}$, and $T_{C D 32}{ }^{\operatorname{dim}} C D 4^{+} T$ cell subsets to $A D C C$ response. Gp120 coated $C D 4^{+} T$ cells from 15 ART-treated and virologically-suppressed PLWH (participants \#8-22, Table S1) were subjected to a flow cytometry-based ADCC assay [35]. In this assay, we used gp120-coated cells and plasma from an HIV-infected person with a high titer of HIV-specific immunoglobulins, which allowed a comparative evaluation of the intrinsic susceptibility of the different subpopulations to NK-mediated killing. Gating of CD32 cells was performed as previously reported in our previous publications $[13,6]$, whereby possible contaminant cell conjugates with monocytes or B cells (defined as $\mathrm{CD} 4^{+} \mathrm{CD} 32^{\text {high }}$ cells), were excluded. Coating efficiency with the recombinant protein gp120 of different cell subsets is shown in Figure S1A, and a representative gating strategy used for the identification of killed cells by ADCC is shown in Figure S1B. Results showed that each CD4 ${ }^{+}$ cell subset had a different susceptibility to autologous NK cells, being $T_{E M}>T_{C M}>$ $T_{C D 20}{ }^{\operatorname{dim}}>T_{N A}>T_{S C M}>T_{C D 32}{ }^{\operatorname{dim}}$ more prone to be killed (ANOVA Friedman test $p=0.0001$ ) (Figure 1A). Overall, most $C D 4^{+} T$ cell subsets were susceptible to $A D C C$, however $T_{C D 32}{ }^{\text {dim }}$ cells, and to a lesser extent $\mathrm{T}_{\mathrm{SCM}}$, showed the highest resistance to ADCC. 
To address if the pattern observed for CD32 cells was exclusive of ART-treated PLWH, we included samples from Elite controllers (EC) and healthy donors (HD). First, we compared the expression of CD32 in CD4 ${ }^{+} \mathrm{T}$ cells in the three cohorts (participants \#1-22, Table S1), following a very stringent flow cytometry gating strategy (Figure S1B). In agreement with previous studies [12, $13,36,37]$, we found that a median of $1.21 \%$ of $C D 4^{+} T$ cells expressed the CD32 receptor, and no statistically significant differences were detected between ART-suppressed, EC participants and HD (Figure S1C). In addition, and in concordance with the study of García et al., but differing from the report of Darcis et al. [12,37], there was no correlation between the HIV-DNA or HIV-RNA levels and the frequency of CD32 expression on CD4 ${ }^{+} \mathrm{T}$ cells (Figure S1D and S1E). We then performed the NKADCC assays. We observed that overall, NK cells from healthy donors were highly efficient at killing the total $\mathrm{CD}^{+} \mathrm{T}$ cell population (Figure 1B) and the different $\mathrm{CD} 4^{+} \mathrm{T}$ subsets (Figure 1C). EC represented a heterogeneous group of individuals, in which no significant differences were detected compared to HD as a total (Figure 1B) or by subset (Figure 1C). In contrast, total CD4 ${ }^{+} \mathrm{T}$ cells (Figure 1B) as well as all subsets from ART-treated PLWH were less susceptible to ADCC-mediated killing than HD (Figure 1C). The altered frequencies, phenotypes, and decreased functions of NK cell subsets reported during HIV infection [38], which are not fully restored by ART [27], could explain the different NK potency observed in these ART-suppressed PLWH. Notably, and regardless of that, NK cells from all cohorts showed a marked impaired capacity to kill $\mathrm{T}_{\mathrm{CD} 32}{ }^{\mathrm{dim}}$ cells (median \% of ADCC of $0.00,34.84$, and 45.01, for ART, EC, and HD, respectively) (Figure 1C and Figure S1F and S1G). We also investigated the potential relationship between the total cell reservoir size in vivo and ADCC activity. We observed a statistically significant inverse correlation between the percentage of ADCC activity against $\mathrm{CD} 4^{+} \mathrm{T}$ cells and the total HIV-DNA reservoir size (Figure 1D) and, in particular, for the $A D C C$ against $T_{C D 32}{ }^{\text {dim }}$ cells (Figure $1 E$ ).

HIV proteins are known to alter the expression of molecules on the infected cells, thereby impacting their recognition and likely the killing mediated by immune cells. Thus, we ought to confirm our results in a more physiological setting using ex vivo infected $\mathrm{CD} 4^{+} \mathrm{T}$ cells. Isolated $\mathrm{CD} 4^{+}$ T cells from ART-suppressed PLWH were infected with HIV $\mathrm{V}_{\mathrm{BaL}}$ or HIV $\mathrm{V}_{\mathrm{NL} 4.3}$, and after 5 days, were subjected to NK natural cytotoxicity (NC) and ADCC assays (participants \#23-29, 36, 44, 46-49 and 51, Table S1). A representative flow gating strategy is shown in Figure 1F. After the ex vivo infection, we observed higher expression of the CD32 molecule in comparison to uninfected cells (Figure S2A and S2B). Moreover, no significant differences between the killing of $T_{C D 32}{ }^{\text {dim }}$ and $T_{C D 32}{ }^{\text {neg }}$ by NC in ART-suppressed PLWH were observed (Figure 1G). However, and in concordance with results in 
Figures $1 \mathrm{~A}$ and $\mathrm{C}, \mathrm{T}_{\mathrm{CD} 32}{ }^{\text {dim }}$ cells from ART-suppressed PLWH were significantly more resistant to ADCC in comparison to their negative cell counterparts (median \% ADCC killing normalized to NC of 19.66 vs 35.85 for $T_{C D 32}{ }^{\text {dim }}$ and $T_{C D 32}{ }^{\text {neg }}$, respectively) (Figure $\mathbf{1 H}$ ). In addition, we observed that the capacity to kill infected $T_{C D 32}{ }^{\text {dim }}$ cells was directly related to the global capacity to kill all infected cells, indicating the essential role of NK potency, in addition to the intrinsic susceptibility of the target cells (Figure S2C). These results show that, regardless of differences between individuals on the overall killing capacity of their NK cells, different subpopulations of infected CD4 ${ }^{+} \mathrm{T}$ cells have distinct intrinsic susceptibility to ADCC responses, being the pool of $\mathrm{CD}^{+} \mathrm{T}$ cells expressing the CD32 molecule more resistant. Moreover, ART-suppressed individuals, most likely due to the existence of impaired NK cells, have a remarkable inability to kill this population of infected cells.

\section{Viral-reactivated cells expressing CD32 from ART-treated PLWH are resistant to NK cell- mediated cytotoxicity.}

Next, we examined if this NK-resistant profile might also affect the latent reservoir after viral reactivation. First, using samples from 9 ART-suppressed PLWH (subjects \#59-67, Table S1), we reactivated $\mathrm{CD}^{+} \mathrm{T}$ cells with different latency-reversing agents (LRA) and applied the Prime Flow RNA (FISH-flow) in situ hybridization (ISH) assay, which allows detecting at a single cell level cells expressing viral RNA, as described before $[39,40]$. The gating strategy is shown in Figure S3A. We observed a higher increase of HIV-RNA ${ }^{+}$cells in the $T_{C D 32}{ }^{\text {dim }}$ fraction compared to $T_{C D 32}{ }^{\text {neg }}$ cells in all conditions and, within $\mathrm{T}_{\mathrm{CD} 32}{ }^{\text {dim }}$ cells, HIV-RNA ${ }^{+}$cells were more frequent after LRA treatment compared to untreated cells (Figure 2A). Upregulation of CD32 expression upon the treatment with several LRAs was evidenced in total $\mathrm{CD}^{+} \mathrm{T}$ cells (Figure S3B). Furthermore, we performed functional NK assays using samples from 22 additional ART-suppressed PLWH after reactivation of the natural HIV reservoir (participants \#68-89, Table S1). First, we evaluated viral reactivation by intracellular p24 (Figure S3C) and successfully observed viral reactivation of dormant HIV in 17 of these samples (Figure 2B). An example of p24 detection using this functional assay is shown in Figure S3D. Also in line with our previous results, we found a higher frequency of viral reactivated cells ( $\mathrm{p} 24^{+}$cells) within the $\mathrm{T}_{\mathrm{CD} 32}{ }^{\operatorname{dim}}$ fraction (Figure $\mathbf{2 C}$ ). After the NK killing assays, viral-reactivated cells, in general, were susceptible to ADCC (Figure 2D, NK + plasma condition). Of note, we used autologous plasma from the same virologically-suppressed PLWH (mean time of undetectable viremia of 60 months [range 25-118]). This is noteworthy since ART treatment may decrease the number of antibodies mediating ADCC [41]. We next evaluated the population of viral-reactivated cells expressing CD32 
in these PLWH. We observed an increase in the expression of CD32 after latency disruption, which constituted a significant fraction of the total pool of viral reactivated cells (Figure 2E). Remarkably, this population was refractory to ADCC and even increased after the ADCC assays (condition with plasma) (Figure 2F). Thus, concordantly with previous results, $T_{C D 32}{ }^{\operatorname{dim}}$ cells showed a higher pattern of ADCC resistance compared to the total HIV-reactivated cells (Figure $\mathbf{2 G}$ ). Altogether, our results show that the latent HIV reservoir expresses CD32 upon viral reactivation with LRAs, and the resulting $\mathrm{T}_{\mathrm{CD} 32}$ dim infected cells are less sensitive to NK-mediated ADCC killing than the whole infected population. Importantly, antibodies endowed with ADCC-triggering capacity are still present in some ART-suppressed PLWH, yet the $\mathrm{T}_{\mathrm{CD} 32}{ }^{\operatorname{dim}}$ population might escape from this immune mechanism.

\section{Infected $\mathrm{T}_{\mathrm{CD} 32}{ }^{\mathrm{dim}}$ cells expressing activating or inhibitory NK ligands are refractory to NK- mediated killing}

To ascertain if differences in receptor-ligand interactions could be responsible for the impaired capacity of the NK cells to kill the $T_{C D 32}{ }^{\text {dim }}$ subset, we studied the expression of MICA/B, ULBP-1, CD155, and HLA-E on the surface of HIV-infected cells after ex vivo infection. Of note, the impact of HIV infection on the expression of many of these ligands is not fully understood and seems to depend on the viral strain and the stage of the viral infection $[42,43]$. Expression of the ligands was assessed by flow cytometry, and the gating strategy used for these analyses is shown in Figure S4A. Overall, we observed that, despite HIV induced higher expression of MICA/B, ULBP-1, and CD155 in infected cells compared to uninfected cells, only a small proportion of infected cells expressed these activating ligands (Figure $\mathbf{3 A}$ and $\mathbf{B}$ ). In contrast, the ligand HLA-E was found to be expressed in a significantly higher proportion of infected cells (median of $12.0 \%$ in HIV-infected cells vs. $9.4 \%$ in uninfected cells, $p=0.002$ ) (Figure $3 C$ and $D$ ). By analyzing infected cells based on their expression of CD32, the same trend was observed for the receptors MICA/B, ULBP1, or CD155 (median 0.6\%, $1.28 \%, 0.37 \%$ for infected $\mathrm{T}_{\mathrm{CD} 32}{ }^{\mathrm{dim}}$ vs. $0.26 \%, 0.09 \%, 0.26 \%$ for the infected $\mathrm{T}_{\mathrm{CD} 32}{ }^{\text {neg }}$ fraction) (Figure 3E and F). However, HLA-E expression was 2-fold significantly higher in HIV-infected $\mathrm{T}_{\mathrm{CD} 32}{ }^{\text {dim }}$ compared to infected $\mathrm{T}_{\mathrm{CD} 32}{ }^{\text {neg }}$ cells (median of $22.2 \%$ and $11.0 \%$ respectively, $p<0.0001$ ) and differences in MFI were also detected (Figure $\mathbf{3 G}$ and $\mathbf{H}$ ). Of note, in the absence of any viral infection, $\mathrm{T}_{\mathrm{CD} 32}{ }^{\mathrm{dim}}$ cells showed intrinsically higher HLA-E expression (Figure S4B).

Next, we ought to determine the expression of activating and inhibitory ligands on the fraction of cells refractory to ADCC after ex vivo infection. We performed both NC and ADCC functional 
201

202

203

204

205

206

207

208

209

210

assays (in cells from participants \#36, 40, 44, 45, 47-49, and 51, Table S1). The infected population $\left(\mathrm{p} 24^{+}\right)$expressing the ligand ULBP-1 was significantly eliminated by both mechanisms, NC and ADCC (Figure 31), however infected cells expressing MICA/B and CD155 were only eliminated by NC (Figure 3J and K). Of note, as previously reported $[25,44]$, cells expressing the molecule HLA-E were particularly resistant to NK-mediated killing (Figure $3 \mathbf{L}$ ). However, infected $\mathrm{T}_{\mathrm{CD} 32}{ }^{\text {dim }}$ cells expressing any of the activating NK ligands were more refractory to both NK-mediated immune responses (Figure 3M-P). Overall, we observed that upon HIV infection, target cells expressing NK-activating ligands were susceptible to natural cytotoxicity mediated by NK cells. However, infected cells expressing HLA-E and, particularly, CD32 ${ }^{\text {dim }}$ cells expressing this molecule and/or the activating ligands MICA/B, ULBP-1, and CD155, were more resistant to NK-mediated killing.

\section{Suboptimal binding of HIV-specific immunoglobulins to CD32 in HIV-infected cells inefficiently} triggers NK cell degranulation

Different hypotheses might help to explain why $T_{C D 32}{ }^{\text {dim }}$ cells are more resistant to $A D C C$. CD32a is a low-affinity receptor for the constant fraction of immunoglobulin G (FcyR-Ila) [45-47], and the expression of this molecule on HIV-infected cells would provide them with a bivalent capacity to interact with immunoglobulins present in plasma, both through their constant fraction (Fc portion) or the variable fragment (antigen-specific). In addition, pentraxins, conserved innate immune molecules involved in infectious processes and inflammation, can bind to CD32 and compete with IgGs [48]. Thus, the engagement of such molecules or immune complexes (IC) to the CD32 Fc receptor might offer a selective advantage by protecting infected cells from HIV-specific ADCCinducing antibodies. Accordingly, we tested if HIV-specific immunoglobulins (Igs) were able to efficiently bind to CD32-expressing cells previously coated with gp120. After incubation with plasma containing HIV-unspecific immunocomplexes (ICS), we found a decrease in the total number of HIVspecific molecules ( $A 32 \mathrm{mAb}$ ) able to bind to $\mathrm{T}_{\mathrm{CD} 32^{\mathrm{dim}}}$ cells (Figure $4 A-B$ ), but such effect was not observed in the $\mathrm{T}_{\mathrm{CD} 32^{\text {neg }}}$ population (Figure $\mathbf{4 C}$ ). A reduction in the total number of cells being recognized by the $A 32 \mathrm{mAb}$ was not observed (Figure S5A-B). This suggests that components present in plasma might bind to the $\mathrm{F} c \gamma \mathrm{R}$ receptor $\mathrm{CD} 32$ representing a steric hindrance and precluding further binding of HIV-specific IgGs. Alternatively, this may indicate competition between CD32 and gp120 for the binding of the same IgG.

We also studied the capacity of ex vivo infected $C D 4^{+} T$ cells expressing $C D 32$ to form ADCCinduced conjugates with NK cells. We observed a significantly higher frequency of $T_{C D 32}{ }^{d i m}-N K$ 
232 doublets compared to $\mathrm{T}_{\mathrm{CD} 32^{\text {neg }}}$ cells (Figure 4D). This could be explained by the inefficient elimination 233 of $T_{C D 32}{ }^{\operatorname{dim}}$ cells by $A D C C$ (as shown in Figure $\mathbf{1 H}$ ), more likely as a consequence of suboptimal 234 antibody-induced immune synapse. This phenomenon has been previously described in HIV235 infected macrophages where increased effector-target cell contact time was associated with 236 relative resistance to cytotoxic T lymphocytes [49]. Indeed, when we tested the capacity of FACS237 sorted and gp120-coated $\mathrm{T}_{\mathrm{CD} 32}{ }^{\operatorname{dim}}$ cells to activate NK cells, we observed that these cells triggered a 238 less potent NK degranulation than their negative counterparts (Figure 4E), while IFN- $\gamma$ was equally 239 induced in both cases (Figure S5C). Overall, our results suggest that potential immune factors 240 present in the plasma that are ligands of CD32 might promote a steric interference that precludes 241 the subsequent binding of HIV-specific antibodies, leading to poor NK activation and therefore 242 resistance to ADCC (as illustrated in Figure $\mathbf{4 F}$ ).

HIV-infected cells expressing CD32 show higher proliferation potential after immune complexes engagement

We next explored the possibility that the engagement of IC to the CD32 molecule might induce cell proliferation, and therefore, contribute to their persistence. To study that, we performed ex vivo infection experiments and measured cell proliferation after IC engagement by flow cytometry. First, we observed that HIV-infected cells, and in particular infected cells expressing CD32 $2^{\text {dim }}$, had higher proliferative potential measured by the expression of Ki67 (median of $6.67 \%$ vs. $4.62 \%$ for

$250 \mathrm{~T}_{\mathrm{CD} 32}{ }^{\operatorname{dim}}$ and $\mathrm{T}_{\mathrm{CD} 32}{ }^{\text {neg }}$, respectively) (Figure 5A and B). Importantly, we observed that the addition of 251 plasma from an HIV-infected patient (containing a high titer of Igs), induced significant proliferation 252 of infected $\mathrm{T}_{\mathrm{CD} 32}{ }^{\mathrm{dim}}$ cells (median of $7.52 \%$ vs. $12.80 \%$ for basal condition and plasma $\mathrm{HIV}^{+}$, 253 respectively), which was abrogated when Fc receptor blockers were added to the culture (Figure 254 5C). Of note, no effect was observed after the addition of plasma from an HIV-negative donor (Figure 255 5C). In contrast, cell proliferation was unchanged in the infected $T_{C D 32}{ }^{\text {neg }}$ population (Figure 5D). 256 Overall, these results suggest that IC present in the plasma of PLWH could contribute to the 257 perpetuation of the $\mathrm{T}_{\mathrm{CD} 32}{ }^{\text {dim }}$ HIV-infected subset by inducing cell proliferation, besides protecting 258 them from ADCC. 
Given the unique properties of $\mathrm{T}_{\mathrm{CD} 32}{ }^{\mathrm{dim}}$ cells, we further explored several strategies to potentiate its elimination by NK cells. First, we tried to directly reinvigorate NK cells from HIV-infected PLWH (\#52-58, Table S1) culturing them with the cytokines IL-15 or IFN- $\alpha$. As expected and previously reported [50], we observed an enhanced performance of NK cells against the total gp120-coated $\mathrm{CD} 4^{+} \mathrm{T}$ cell population when treated with IL-15, and to a lesser extent with IFN- $\alpha$. However, neither IL-15 nor IFN- $\alpha$ was able to enhance the $A D C C$ response against $T_{C D 32}{ }^{\operatorname{dim}}$ infected cells (Figure 6A). Moreover, we showed before that $\mathrm{T}_{\mathrm{CD} 32}{ }^{\operatorname{dim}}$ cells express a remarkable higher levels of the NK-ligand HLA-E. Thus, using ex vivo infected cells, we performed functional assays blocking the interaction of HLA-E with its cognate receptors on NK cells by adding specific antibodies against HLA-E (participants \#38, 44, and 46-51, Table S1). In general, the NC response was not enhanced after HLA-E blocking, either in the whole population of infected cells $\left(\mathrm{p} 24^{+}\right)$(Figure 6B) or in the $T_{C D 32}{ }^{\operatorname{dim}}$ subpopulation (Figure 6C). However, we observed a more potent ADCC immune response against all infected cells after blocking HLA-E (Figure 6D), including the infected $\mathrm{T}_{\mathrm{CD} 32}{ }^{\operatorname{dim}}$ pool (Figure 6E). Remarkably, the use of an isotype control antibody also significantly enhanced the killing of all infected cells (Figure 6D and E), evidencing the non-specificity of the anti-HLA-E antibody at enhancing ADCC. Thus, blocking the interaction of HLA-E with its receptors in NK cells did not specifically reverse the intrinsic immune resistance of $\mathrm{T}_{\mathrm{CD} 32}{ }^{\mathrm{dim}}$ cells.

\section{DISCUSSION}

The presence of cellular and anatomical viral reservoirs, not susceptible to ART or antiviral immune responses, is the main barrier to cure the HIV infection. Thus, elucidating how these reservoirs are maintained for prolonged periods of time represents an important step towards the cure of HIV. Remarkably, we postulate a pivotal role of ADCC-NK in shaping the HIV reservoir during ART, being a novel route to avoid NK cell effector immunity by HIV-reservoir cells. We found that the expression of the molecule CD32 on productively HIV-infected cells is associated with a reduced susceptibility to ADCC activity by NK cells. Furthermore, when infected ex vivo, these cells express higher levels of the molecule HLA-E, which also limits NK-mediated killing. Last, upon interaction with immune complexes, their capacity to bind to HIV-specific antibodies decreases, while gaining the potential to proliferate. Considering that a significant proportion of HIV-latently infected cells expressed CD32 upon viral reactivation, as shown here and reported before [40], these cells will 
291 indeed benefit from these described NK immune evasion mechanisms. Altogether, these factors 292 may greatly contribute to perpetuating the persistence of the cell reservoir.

293 One of the main obstacles in the HIV cure field has been the lack of reliable markers to uniquely 294 identify persistently infected cells. Amongst proposed molecules, we find immune checkpoint 295 inhibitors such as PD-1 [51], the B cell surface marker CD20 [19], CD30 [20], or, more recently, a 296 combination of several receptors [8]. However, the Fcy receptor CD32 is perhaps one of the most 297 promising HIV reservoir markers, since it is expressed during latent $[9,12]$ and transcriptionally298 active infection $[13,15,16,52]$; it has been localized in main tissue reservoirs such as the cervical 299 tissue [6], lymph nodes [13, 16] and the gastrointestinal tract [15] and, in some cases, it has been 300 associated with a very prominent enrichment for $\operatorname{HIV}$ DNA $[6,9,12]$. This molecule is typically 301 expressed on myeloid cells or platelets, in which its function has been extensively studied [47]. While 302 CD32 expressed on $\mathrm{CD}^{+} \mathrm{T}$ cells is fully functional $[36,53]$, many questions remain unknown 303 regarding the expression dynamics and function, in particular during HIV pathogenesis. In a recent study with SIV-infected non-human primates, $\mathrm{CD} 32^{+} \mathrm{CD} 4^{+} \mathrm{T}$ cells were strongly increased in LNs, spleen, and intestine during SIV mac infection, were enriched in markers often expressed on HIV infected cells, and contained higher levels of actively transcribed SIV RNA [52]. Consistent with 307 previous reports [13, 37], we detected dim levels of CD32 on CD4 ${ }^{+} T$ cells which increased upon ex 308 vivo HIV infection or reactivation. This result is in line with a recent publication showing the ability 309 of cells expressing CD32 to reactivate latent HIV [12]. Moreover, tissue-resident CD4 ${ }^{+} \mathrm{T}$ cells with 311 this fraction of cells was intrinsically enriched for the expression of molecules related to HIV 312 susceptibility and long-term maintenance [6]. In this sense, cell proliferation is one of the most 313 important mechanisms of cell reservoir maintenance in long-term ART-suppressed PLWH $[3,54,55]$. 314 Importantly, we show that CD32-expressing cells had a higher proliferative potential in response to 315 IC. Inherent proliferative capacity of this subset has recently been reported in a study demonstrating $316 \mathrm{CD}^{+} \mathrm{T}$ cell activation upon CD32 ligation with antibodies or aggregated IgG [36]. Thus, 317 understanding the mechanisms by which HIV-infected cells expressing CD32 are maintained in the 318 human body could significantly advance the search for an HIV cure.

319 NK cells are key players in the defense against many pathogens, including HIV, being not only 320 one of the first lines of protection but also essential modulators of the adaptive immune responses. 321 Therefore, the acquisition of resistance mechanisms to any of the NK effector functions may 
322 contribute to pathogen survival and, in the case of HIV, favor conditions for viral persistence. In this

323 sense, it has been reported that NK cell immune pressure leads to viral sequence evolution [56], and

324 HLA-mediated immune resistance mechanisms have been previously identified in productively HIV-

325 infected cells $[57,58]$. Moreover, resistance of reservoir cells to HIV-specific cytotoxic T cells has

326 also been reported $[59,60]$, suggesting that viral persistence might be facilitated not only by cell

327 proliferation mechanisms in the absence of viral antigen expression, but also by avoiding immune-

328 mediated killing. NK cells recognize IgG-viral protein complexes, namely immune complexes, on

329 infected cells via FcyRs to mediate ADCC, which is a potent mechanism to eliminate virally infected

330 cells [61]. However, HIV has developed several strategies to evade this immune response. For

331 instance, Vpu reduces the presence of viral antigens susceptible to recognition by antibodies on the

332 surface of infected cells [62]. This accessory protein is responsible for the decreased expression of

333 tetherin, a cellular host restriction factor that retains HIV virions on the cell surface, and therefore

334 diminishes ADCC responses [62]. Moreover, Vpu and Nef downregulate CD4 expression on infected

335 cells, preventing its interaction with Env trimers, which subsequently impedes the binding of ADCC-

336 antibodies [63]. Overall, a proper antibody-induced immune synapse with NK cells is required to

337 elicit a potent ADCC immune response, which depends on many factors, such as spatial

338 configuration, valence of the antibody-epitope binding, antibody conformation, and the resulting

339 size of the immune complex [64]. In our study, we show that the interaction of $\mathrm{T}_{\mathrm{CD} 32}{ }^{\mathrm{dim}}$ cells with IC

340 present in plasma lead to suboptimal binding of HIV-specific antibodies, limiting ADCC. These results

341 suggest that potential Igs, and likely other immune mediators such as pentraxins [48], might

342 contribute to maintaining HIV-reservoir cells through the interaction with the CD32 receptor,

343 conferring a diminished susceptibility to ADCC activity, which intrinsically requires the presence of

344 immune complexes. This is not the first non-desirable effect of Igs identified in the context of an

345 infectious disease; an antibody-dependent enhancement of infection has been extensively reported

346 for Dengue infection [65]. The intrinsic nature of antibodies, with an antigen-binding fragment (Fab)

347 and a crystallizable fragment $(\mathrm{Fc})$ as separate functional domains, adds complexity to the multiple

348 functions and interactions that ultimately contribute to modulate immunity [66]. Importantly,

349 increased knowledge on the Fc effector functions of IgG antibodies is greatly contributing to the

350 design of new therapeutic strategies based on Fc-optimized monoclonal antibodies to promote

351 effective Fc effector activities [67]. In fact, a major line of research in the field currently focuses on

352 the use of broadly neutralizing antibodies (bNAbs) as a therapeutic or prophylactic treatment for

353 HIV infection, relying on the efficient blocking of the virus and the triggering of potent immune 
354 effector responses, for example, through FcyR interactions [68, 69]. The potentiation of the NK 355 activity with these new approximations may help to overcome the intrinsic resistance of infected 356 cells expressing the CD32 receptor.

357 Furthermore, as previously reported by others, HIV infection maintains or even increases the 358 expression of the non-classical HLA-E molecule, which may contribute to the inhibition of the NK 359 cytotoxic response [44, 70]. Interestingly, we found enrichment of HLA-E expression in the pool of 360 HIV-infected cells expressing CD32, which could tilt the balance towards NK inhibition, therefore, 361 conferring an additional advantage to survive. HLA-E can interact with NK cells through two 362 receptors: NKG2A (inhibitory) and NKG2C (activating) [71]. Of note, the affinity of the NKG2A protein 363 for HLA-E is higher than that of NKG2C [72]. Further, NKG2A ${ }^{+}$NK cells do not typically express 364 NKG2C, thus representing a pool of NK cells that can potentially be restricted by HLA-E [73]. The 365 effect of HLA-E suppressing immune responses has been shown in several settings, including in the 366 context of senescence or tumors, where blocking its interaction reinvigorated effector functions [24, 367 25]. In addition, HLA-E has been shown to impair ADCC against HIV-expressing cells, while impeding 368 its interaction with NK cells improved the elimination of target cells [44]. Our results showed an 369 enhancement of NK-mediated killing of CD32 $2^{\text {dim }}$ HIV-infected cells by ADCC when HLA-E was blocked. 370 However, this potentiation was not specific, since the isotype control induced a similar effect. The 371 killing of aberrant cells mediated by NKs is determined by a complex balance of signaling received 372 through their multiple receptors which interact with molecules on the surface of undesired cells 373 [22]. Thus, other molecules expressed by $T_{C D 32}{ }^{\text {dim }}$ cells may be contributing to elevating the threshold 374 for NK activation needed to eliminate this cell subpopulation in these individuals.

375 In recent years, major efforts have focused on the identification of compounds to reactivate 376 persistent HIV from its dormant state, with the ultimate goal to eliminate viral infection However, 377 it has become clear that the stimulation of the immune system is also a mandatory step for the 378 elimination of persistent HIV [74]. These approaches, known as shock and kill, have shown little 379 efficacy in several clinical trials in terms of HIV reservoir reactivation and the reduction of its size 380 [75]. This failure has been attributed to both, a poor capacity of the different LRAs to reactivate all 381 latent HIV [40], together with the dysfunction of the immune cells to kill viral reactivated cells [76]. 382 Our results show that the magnitude of the killing also depended on the potency of the NK cells, 383 where NK cells from ART-suppressed PLWH with higher total HIV reservoir size were functionally 384 impaired. These results are in agreement with previous reports showing that HIV chronic infection 
has a deleterious effect on NK cell function, which is not completely restored despite ART [26, 27]. To reinvigorate NK function in these subjects we treated NK cells with the cytokines IL-15 or IFN- $\alpha$. Despite a clear immune effect on the total infected population, we did not increase the killing of $T_{C D 32}{ }^{\text {dim }}$ cells. A plausible explanation would be the upregulation of NKG2A on NK cells mediated by these cytokines $[77,78]$, which would further limit the killing of cells already expressing high levels of HLA-E, such as $T_{C D 32}{ }^{\text {dim }}$ cells.

A potential limitation of our study is the decline of HIV-specific antibody levels in ART-treated PLWH $[41,79]$. Despite this decline, functional ADCC activity mediated by NK cells remains detectable in long-term virally-suppressed individuals [41]. Of relevance, we show here the existence of functional ADCC activity using plasma samples from virologically-suppressed PLWH. These antibodies induced significant NK-mediated elimination of the total pool of reactivated latently infected cells in many samples, although the subpopulation expressing CD32 was more resistant. Thus, our results suggest that, after viral reactivation, levels of HIV antigen expression susceptible to recognition by immune cells such as NK may be induced. Also, that ADCC could play a significant role in HIV persistence, highlighting the need for new strategies directed to impact resistant reservoir cells such as $\mathrm{T}_{\mathrm{CD} 32}{ }^{\mathrm{dim}}$ cells. However, the limited number of these cells observed in vivo in ART-treated PLWH restrains their study. In this sense, the few viral-reactivated cells detected after LRA treatment from the natural latent reservoir impede an extensive assessment of infected cells expressing CD32. Further, it is unclear which is the dynamic of CD32 expression on individual cells after viral reactivation and if changes on its expression would affect ADCC activity. Last, it is possible that infected $\mathrm{T}_{\mathrm{CD} 32}{ }^{\mathrm{dim}}$ cells are susceptible to cytotoxic $\mathrm{CD} 8^{+} \mathrm{T}$ lymphocytes. Further research on these aspects are warranted. However, based on our results, we may speculate that this sort of immune resilient infected cells in vivo could certainly contribute to the source of viral rebound when ART is interrupted, or they could initiate a low level of viral replication in tissues where drugs are not able to fully penetrate. Moreover, their proliferative capacity in response to IC situates these cells as candidates for cells supporting cellular proliferation, one of the main mechanisms that perpetuate HIV reservoirs in vivo $[80,81]$. 


\section{Materials and Methods}

\section{Ethics statement and patient samples}

PBMCs from PLWH were obtained from the HIV unit of the Hospital Universitari Vall d'Hebron in Barcelona, Spain. Study protocols were approved by the corresponding Ethical Committees

419 (Institutional Review Board numbers PR(AG)270/2015 and PR(AG)39/2016). PBMCs from healthy 420 donors were obtained from the Blood and Tissue Bank, Barcelona, Spain. All subjects recruited to 421 this study were adults who provided written informed consent. Samples were completely 422 anonymous and untraceable and were prospectively collected and cryopreserved in the Biobank 423 (register number C.0003590). Information on plasma viral loads, CD4 ${ }^{+}$T cell counts, and time on ART 424 from suppressed PLWH is summarized in Table S1.

PBMCs were obtained from PLWH and uninfected donors by Ficoll-Paque density gradient centrifugation and cryopreserved in liquid nitrogen. PBMCs were cultured in RPMI medium (Gibco) 428 supplemented with 10\% Fetal Bovine Serum (Gibco), $100 \mu \mathrm{g} / \mathrm{ml}$ streptomycin (Fisher Scientific), and $100 \mathrm{U} / \mathrm{ml}$ penicillin (Fisher Scientific) (R10 medium), and maintained at $37^{\circ} \mathrm{C}$ in a $5 \% \mathrm{CO}_{2}$ incubator. For RNA FISH-flow assays, fresh PBMCs were obtained from a whole blood donation (400ml) from PLWH by Ficoll-Paque density gradient centrifugation and $\mathrm{CD}^{+} \mathrm{T}$ cells were immediately isolated 432 and used without previous cryopreservation.

All plasmids needed for the generation of viral stocks and delta molecular clones were obtained through the NIH AIDS Reagent Program. Viral stocks were generated by transfection of 293T cells

436 titrated in TZMbl cells using an enzyme luminescence assay (britelite plus kit; PerkinElmer) as 437 described previously [82]. BaL gp120 recombinant protein was obtained through the NIH AIDS 438 Reagent Program. The A32 antibody was obtained through the AIDS Research and Reference 439 Program, NIAID, NIH (Cat\#11438) from Dr. James E. Robinson [83]. Interleukin-2 (IL-2) was obtained 440 from the Vall d'Hebron Hospital pharmacy. The pan-caspase inhibitor named Q-VD-OPh (quinolyl441 valyl-0-methylaspartyl-[-2,6-difluorophenoxy]-methyl ketone was purchased from Selleckchem. 
PBMCs from ART-suppressed PLWH, elite controllers, or uninfected donors were thawed and rested overnight in R10 medium. To exclude monocytes, PBMCs were cultured in a lying flask and adherent cells were discarded the next day. $\mathrm{CD}^{+} \mathrm{T}$ cells and NK cells were isolated from cryopreserved PBMCs using commercial kits (MagniSort Human CD4 ${ }^{+} \mathrm{T}$ Cell Enrichment; Affymetrix, and MagniSort ${ }^{\mathrm{TM}}$ Human NK cell Enrichment; eBioscience). Two rounds of cell separation were performed to maximize the purity of the cells (overall purity $>85 \%$ ). $C D 4^{+} \mathrm{T}$ cells were stained with the membrane lipid marker PKH67 (Sigma-Aldrich) following the manufacturer's instructions, for the stable identification of these target cells, and then coated with $1 \mu \mathrm{g}$ of recombinant gp120 protein for $1 \mathrm{~h}$ at RT. A pool of uncoated cells was used as a negative control. After coating, target cells were extensively washed in ice-cold R10 medium and dispensed in U-bottom 96-well plates (100,000 cells per well, 10 wells per condition). After that, $C D 4^{+} \mathrm{T}$ cells were incubated for 15 mins with plasma (1:1,000 dilution) from a viremic (high viral load in blood) $\mathrm{HIV}^{+}$patient. Then, NK effector cells were added at 1:1 target/effector ratio. Plates were centrifuged at 400xg for 3 mins and incubated for $4 \mathrm{~h}$ at $37{ }^{\circ} \mathrm{C}$ and $5 \% \mathrm{CO}_{2}$. After incubation, cells were collected in FACs tubes, washed with staining buffer (PBS 3\% FBS), and stained with anti-CCR7-PE-CF594 (150503, Becton Dickinson) for 30 mins at $37^{\circ} \mathrm{C}$. Next, cells were washed and stained with anti-CD3-AF700 (SK7, Biolegend), anti-CD45RO-BV605 (UCHL1, Biolegend), anti-CD32-PE-Cy7 (FUN-2, Biolegend), antiHLA-DR-BV711 (L243, Biolegend), anti-CD20-BV786 (2H7, Biolegend), and anti-CD95-PE-Cy5 (DX2, Becton Dickinson) for 20 mins at RT. Finally, cells were washed with staining buffer (PBS 3\% FBS) and fixed with PFA (2\%). Flow cytometry particles for absolute cell counting $\left(5 * 10^{4} / \mathrm{ml}\right.$ ) (AccuCount Blank 5.0-5.9 $\mu \mathrm{m}$, Cytognos) were added. Samples were acquired on an LSR Fortessa flow cytometer (Becton Dickinson) and analyzed using FlowJo V10 software. We calculated the \% of killing as the number of cells that disappeared in each population, following the next formula:

$$
\% \text { ADCC }=100-\frac{\text { Cells }(\text { gp } 120)}{\text { Cells }(\text { no gp } 120)} * 100
$$

Control cells non-coated with gp120 (no gp120 condition) but incubated with plasma allowed us to properly measure ADCC responses and rule out a possible non-specific activation of the NK cells mediated by the plasma.

In some experiments, we assessed the capacity of NK cells to perform ADCC after being stimulated with IFN- $\alpha$ or IL-15 (Miltenyi Biotec). In such cases, PBMCs were stimulated overnight with 5,000

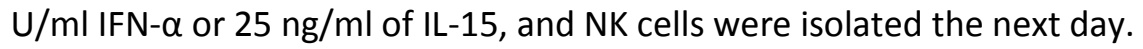


$\mathrm{CD}^{+} \mathrm{T}$ cells were coated with recombinant gp120 as described above and then stained with anti(UCHL1, Biolegend), anti-CD32-PE-Cy7 (FUN-2, Biolegend), anti-HLA-DR-BV711 (L243, Biolegend), anti-CD20-BV786 (2H7, Biolegend) and anti-CD95-PE-Cy5 (DX2, Becton Dickinson) antibodies. Next, cells were incubated for 20 mins at RT with $5 \mu \mathrm{g} / \mathrm{ml}$ of A32 antibody (which binds to gp120 HIV protein). Then, cells were stained for 20 mins at RT with an anti-human FITC-labelled secondary antibody (dilution 1:100) (Thermo Fisher) for A32 detection. Samples were acquired on an LSR Fortessa flow cytometer and data analyzed using FlowJo.

\section{Binding assessment of HIV-specific antibodies to $\mathrm{T}_{\mathrm{CD} 32}{ }^{\text {dim }}$ cells}

Isolated $\mathrm{CD}^{+} \mathrm{T}$ cells from uninfected donors were coated with gp120 recombinant protein. To detect if IC might result in steric hindrance precluding the binding of HIV-specific Igs to the $T_{C D 32}{ }^{d i m}$ subset, we labeled the gp120-specific antibody (A32 mAb) with Allophycocyanin (APC) following the manufacturer instructions (Zenon Human IgG labeling kit, Invitrogen) and measured cell binding by plasma containing high titer of non-HIV specific immune complexes (dilution 1,000) for 20 mins at RT. After, cells were incubated with A32-APC $(3.3 \mu \mathrm{g} / \mathrm{ml})$ for 25 minutes and labeled with anti-CD3AF700 (SK7, Biolegend) and anti-CD32-PE-Cy7 (FUN-2, Biolegend). After, cells were washed once with PBS and stained with LIVE/DEAD Fixable Violet Viability (Invitrogen) for 20 mins at RT. Finally, cells were washed with PBS and fixed with PFA (2\%). Samples were acquired on an LSR Fortessa flow

493 cytometer and data analyzed using FlowJo.

\section{ADCC-mediated NK cell activation of isolated $\mathrm{T}_{\mathrm{CD} 32}{ }^{\mathrm{dim}}$ cells.}

For cell sorting experiments, 100 million PBMCs from healthy donors were stained with LIVE/DEAD violet viability (Invitrogen) for 20 mins at RT. After washing, cells were surface stained with anti-CD3-PerCP (SK7; Becton Dickinson), anti-CD56-FITC (B159, Becton Dickinson), anti-CD32PE-Cy7 (FUN-2, Biolegend) and, anti-CD4-BV605 (RPA-T4, Becton Dickinson) antibodies for 20 mins at RT. Cells were then washed and immediately sorted using a BD FACSAria Cell Sorter. We sorted

501 Then, we performed the ADCC assay and measured NK cell activation by flow cytometry. Purity of the cells was $>97 \%$ in all cases. 
Sorted $T_{C D 32}{ }^{\text {dim }}$ and $T_{C D 32}$ cells were coated with recombinant gp120 as described above and incubated with plasma from a viremic HIV-infected patient at 1:1,000 dilution 15 minutes before the addition of NK cells (ratio 1:2). Co-cultures were maintained for $4 \mathrm{~h}$ in a 96 -well plate at $37{ }^{\circ} \mathrm{C}$ and $5 \% \mathrm{CO}_{2}$. NK cytotoxicity was assessed by measurement of CD107a and IFN- $\gamma$. As a positive control, we included NK cells cultured with $10 \mathrm{ng} / \mathrm{ml}$ PMA plus $1 \mu \mathrm{M}$ ionomycin, and as a negative control, NK cells were cultured without any stimulus. CD107a-PE-Cy5 (H4A3; Beckton Dickinson), BD GolgiPlug Protein Transport Inhibitor (Becton Dickinson) and, BD GolgiStop Protein Transport Inhibitor containing monensin (Becton Dickinson) were also added to each well at the recommended concentrations at the beginning of cell culture. After incubation, cells were washed and stained with a viability dye (LIVE/DEAD Fixable Violet dead cell stain; Invitrogen). Cells were then stained with anti-CD56-FITC (B159; Becton Dickinson), anti-CD32-PE-Cy7 (FUN-2, Biolegend) and, anti-CD4-BV605 (RPA-T4, Becton Dickinson) antibodies for 20 mins at RT. After that, cells were washed and fixed and permeabilized with Fixation/Permeabilization Solution (Becton Dickinson) for 20 mins at 4 으, washed with BD Perm/Wash buffer, and stained with anti-IFN- $\gamma$ AF700 (Life technologies) for 30 mins at $4 \stackrel{\circ}{\circ}$. After washing, cells were fixed with PFA (2\%) and acquired on an LSR Fortessa flow cytometer (Becton Dickinson). Flow cytometry particles for absolute cell counting $\left(5^{*} 10^{4} / \mathrm{ml}\right.$ ) (AccuCount Blank 5.0-5.9 $\mu \mathrm{m}$, Cytognos) were added. Data were analyzed using FlowJo V10 software.

\section{Ex vivo infection of unstimulated PBMCs}

PBMCs from ART-suppressed PLWH or healthy donors were thawed and incubated overnight in R10 medium containing $40 \mathrm{U} / \mathrm{ml} \mathrm{IL-2}$. The next day, $\mathrm{CD}^{+} \mathrm{T}$ cells were isolated using a commercial kit (MagniSort Human $\mathrm{CD}^{+} \mathrm{T}$ Cell Enrichment; Affymetrix) and infected by incubation for $4 \mathrm{~h}$ at $37^{\circ} \mathrm{C}$ with 156,250 or $2,500 \mathrm{TCID}_{50}\left(50 \%\right.$ tissue culture infectious dose) of HIV $\mathrm{NL4.3}_{3}$ or $\mathrm{HIV}_{\mathrm{BaL}}$ viral strains, respectively. In some experiments, cells were infected by spinoculation at $1200^{*} \mathrm{~g}$ for $2 \mathrm{~h}$ at 37 oc ( $\mathrm{TCID}_{50}$ of 78,125 and 625 for $\mathrm{HIV}_{\mathrm{NL} 4.3}$ and $\mathrm{HIV}_{\mathrm{BaL}}$ ). Cells were then washed twice with PBS and cultured at $1 \mathrm{M} / \mathrm{ml}$ in a 96-well plate round-bottom with R10 containing $100 \mathrm{U} / \mathrm{ml}$ of IL-2 for the next 5 days. The resulting HIV-infected $C D 4^{+} T$ cells were used for different experiments listed below.

\section{Phenotyping of HIV-infected CD4 ${ }^{+}$T cells}

HIV-infected cells were stained with LIVE/DEAD AQUA viability (Invitrogen) for 30 mins at RT. After washing once with staining buffer, cells were stained with anti-ULBP1-PerCP (170818, R\&D 
533 Systems), anti-CD32-PE-Cy7 (FUN-2, Biolegend), anti-CD3-PE-Cy5 (UCHT-1, Biolegend), anti-CD4-

534 AF700 (RPA-T4, Becton Dickinson), anti-HLA-E-APC (3D12, Biolegend), anti-CD155-BV786 (TX24,

535 Becton Dickinson) and anti-MIC A/B -BV605 (6D4, Becton Dickinson) antibodies for 20 mins at RT.

536 Cells were then fixed and permeabilized with Fixation/Permeabilization Solution (Becton Dickinson)

537 for 20 mins at 4 으, washed with BD Perm/Wash buffer, and stained with anti-p24-PE (Beckman

538 Coulter) for 20 mins on ice and 20 mins at RT. After washing with BD Perm/Wash buffer, cells were

539 fixed with PFA (2\%). Samples were acquired on an LSR Fortessa flow cytometer and data analyzed

540 using FlowJo V10 software. Gating was performed according to the different FMO controls.

\section{Assessment of the proliferative potential of $\mathrm{T}_{\mathrm{CD} 32}{ }^{\operatorname{dim}}$ cells}

HIV-infected cells were incubated with plasma from a viremic $\mathrm{HIV}^{+}$patient or plasma from an

543 uninfected healthy donor (dilution $1: 1,000$ ) for $4 \mathrm{~h}$ at $37{ }^{\circ} \mathrm{C}$ and $5 \% \mathrm{CO}_{2}$. Additionally, a pool of HIV-

544 infected cells previously treated with an Fc receptor blocker (human Fc block, Becton Dickinson) was

545 used as a control. After incubation, cells were washed and stained with LIVE/DEAD Fixable Violet

546 Viability (Invitrogen) for 20 mins at RT. Next, cells were washed with staining buffer and stained with

547 anti-CCR7-PE-CF594 (150503, Becton Dickinson) for 30 mins at 37 ㅇ․ After washing, cells were

548 stained with anti-CD56-FITC (B159, Becton Dickinson), anti-CD3-AF700 (SK7, Biolegend), anti-

549 CD45RO-BV605 (UCHL1, Biolegend), anti-CD32-PE-Cy7 (FUN-2, Biolegend), anti-CD20-BV786 (2H7,

550 Biolegend), anti-CD95-PE-Cy5 (DX2, Becton Dickinson), and anti-CD4-APC (OKT4, Biolegend) for 20

551 mins at RT. Cells were then fixed and permeabilized with Fixation/Permeabilization Solution (Becton

552 Dickinson) for 20 mins at 4으, washed with BD Perm/Wash buffer and stained with anti-p24-PE

553 (KC57, Beckman Coulter) for 20 mins on ice and, during the additional 30 mins at RT an anti-Ki67-

554 BV510 (B56, Becton Dickinson) was added. Finally, cells were washed and fixed with PFA (2\%).

555 Positive cells for the ki67 marker were determined according to FMO controls. Samples were

556 acquired in an LSR Fortessa flow cytometer (Becton Dickinson) and analyzed with FlowJo V10 557 software.

\section{$558 \quad$ Natural cytotoxicity and ADCC NK-based assays}

5595 days after infection, HIV-infected cells were placed in a 96 round bottom well plate at 100,000 560 cells/well (ten replicates). Autologous NK cells, previously isolated by negative selection using 561 magnetic beads (MagniSort ${ }^{T M}$ Human NK cell Enrichment Kit, eBioscience), from PBMCs thawed the 562 day before the co-culture, were added at 1:1 ratio. For the study of the ADCC response, plasma from 
a viremic HIV-infected patient containing a mix of antibodies targeting different HIV epitopes was added at 1:1,000 dilution to HIV-infected cells 15 minutes before the addition of NK cells. After, the plate was centrifuged at $400^{*} \mathrm{~g}$ for 3 minutes to facilitate cell contact and then incubated at $37 \stackrel{\circ}{\mathrm{C}}$ with $5 \% \mathrm{CO}_{2}$ for $4 \mathrm{~h}$. After, cells were collected in FACs tubes, washed with PBS, and stained with LIVE/DEAD Fixable Violet Viability (Invitrogen) for 20 mins at RT. Next, cells were washed with staining buffer and stained first with anti-CCR7-PE-CF594 for 30 mins at 37 으, and after an additional wash, with anti-CD56-FITC (B159, Becton Dickinson), anti-CD3-AF700 (SK7, Biolegend), anti-CD45RO-BV605 (UCHL1, Biolegend), anti-CD32-PE-Cy7 (FUN-2, Biolegend), anti-CD20-BV786 (2H7, Biolegend), anti-CD95-PE-Cy5 (DX2, Becton Dickinson), and anti-CD4-APC (OKT4, Biolegend) antibodies for 20 mins at RT. Cells were then fixed and permeabilized with Fixation/Permeabilization Solution (Becton Dickinson) for 20 mins at $4 \stackrel{\circ}{ }$ C, washed with BD Perm/Wash buffer, and stained with anti-p24-PE (KC57, Beckman Coulter) for 20 mins on ice and 20 mins at RT. Finally, cells were washed and fixed with PFA (2\%). Flow cytometry particles for absolute cell counting $\left(5^{*} 10^{4} / \mathrm{ml}\right.$ ) (AccuCount Blank 5.0-5.9 $\mu \mathrm{m}$, Cytognos) were added. We also included a non-infected condition. Gating of the CD32 subpopulation was done according to FMO controls. Samples were acquired in an LSR Fortessa flow cytometer and analyzed with FlowJo software. The percentage of ADCC killing was determined by calculating the number of cells that disappeared in each infected population, normalizing numbers to the condition of HIV-infected $\mathrm{CD}^{+}{ }^{+} \mathrm{T}$ cells plus NK cells (in the absence of plasma). The $\mathrm{NC}$ response was quantified as the reduction in the \% of p24 HIV protein in $\mathrm{T}_{\mathrm{CD} 32^{\text {neg }}}$ and $\mathrm{T}_{\mathrm{CD} 32^{\text {dim }}}$ subsets, in comparison to the \% of p24 in these subsets in basal conditions in the absence of NK cells. In the experiments assessing the effect of blocking HLA-E, HIV-infected cells were previously incubated with $10 \mu \mathrm{g} / \mathrm{ml}$ of anti-human HLA-E (clone 3D12, Biolegend) antibody for 20 mins at RT, or with an isotype control mouse IgG1 (Becton Dickinson). In these assays, cells were first stained with AQUA viability (Thermo Fisher), then with a panel of surface antibodies: anti-ULBP1-PerCP (170818, R\&D Systems), anti-CD56-FITC (B159, Beckton Dickinson), anti-CD32-PE-Cy7 (FUN-2, Biolegend), anti-CD3-PE-Cy5 (UCHT-1, Biolegend), anti-CD4-AF700 (RPA-T4, Becton Dickinson), antiHLA-E-APC (3D12, Biolegend), anti-CD155-BV786 (TX24, Becton Dickinson), anti-MIC A/B -BV605 (6D4, Becton Dickinson); and finally intracellularly stained with anti-p24-PE (KC57, Beckman Coulter) as previously described.

Cell conjugates were identified by flow cytometry and quantified as follow: live lymphocytes (isolated $\mathrm{CD}^{+} \mathrm{T}$ and $\mathrm{NK}$ cells) were gated after excluding contamination with B cells and monocytes. Then, HIV-infected cells were identified as $\mathrm{p}^{2} 4^{+}$cells in $\mathrm{CD}^{+}$cells, from which we selected separately 
595 both, CD32 ${ }^{\text {dim }}$ and $C D 32^{\text {neg }}$ cells. From CD32 ${ }^{\text {dim }}$ and $C D 32^{\text {neg }}$ populations we gated cell doublets by 596 side scatter signals, and then we determined the fraction of cell doublets composed by CD4 ${ }^{+} \mathrm{T}$ cells 597 (CD3+ and CD32 ${ }^{\text {dim }}$ or CD32 ${ }^{\text {neg }}$ ) and NK cells (identified as $\mathrm{CD}^{+} 6^{+}$cells).

\section{Detection of cells expressing HIV-1 RNA by the RNA FISH-flow assay}

PBMCs from nine ART-treated PLWH were obtained from a whole blood donation $(400 \mathrm{ml})$ and

$600 \mathrm{CD}^{+} \mathrm{T}$ cells were isolated by a negative selection kit (MagniSort Human $\mathrm{CD}^{+} \mathrm{T}$ Cell Enrichment; 601 eBioscience). At least $6 \times 10^{6}$ of freshly-isolated $C D 4^{+} T$ cells were studied per condition, being 602 subjected to viral reactivation with different LRAs (Ingenol and Romidepsin), and including the 603 positive (PMA plus ionomycin) and negative controls (medium condition). Before viral reactivation, 604 cells were pre-incubated with the pan-caspase inhibitor Q-VD-OPh (Selleckchem) for $2 \mathrm{~h}$. In addition, 605 to prevent new rounds of viral infection during HIV reactivation, the cells were treated with LRAs in 606 the presence of Raltegravir (1uM) for $22 \mathrm{~h}$. Afterward, the RNA FISH-flow assay was performed 607 according to the manufacturer's instructions (Human PrimerFlow RNA Assay, eBioscience) with 608 some modifications as previously described [39]. Briefly, after antibody staining and cell fixation and 609 permeabilization, cells were ready for hybridization with a set of 50 probes spanning the whole GagPol HIV mRNA sequence (bases 1165 to 4402 of the HXB2 consensus genome). Next, the cells were

611 subjected to amplification signal steps, and HIV RNA was detected using Alexa Fluor 647-labeled 612 probes. In these experiments, for surface antigen labeling, anti-human CD3 (AF700, Biolegend), anti613 human CD32 (PE-Cy7, Biolegend), anti-human CD20 (BV785, Biolegend), and anti-human HLA-DR 614 (BV711, Biolegend) antibodies were used, and cell viability analyzed with violet viability dye 615 (Invitrogen). Samples were analyzed with the LSR Fortessa flow cytometer, and the results were 616 analyzed with FlowJo v10 software. in R10 medium with Q-VD-OPh (Selleckchem) for $2 \mathrm{~h}$ in the presence of Raltegravir ( $1 \mu \mathrm{M})$, Darunavir

$620(1 \mu \mathrm{M})$, and Nevirapine $(1 \mu \mathrm{M})$ to prevent new rounds of viral infection. After $2 \mathrm{~h}$, PMA plus 621 ionomycin (PMA $81 \mathrm{nM}$; ionomycin $1 \mu \mathrm{M}$ ) were added to the cell culture as a latency reversal agent 622 and left for $18 \mathrm{~h}$ to reactivate latent HIV. After viral reactivation, cells were subjected to cytotoxicity 623 and ADCC assays as previously described here. For these experiments, we used the autologous 624 plasma from each patient. After, cells were stained with LIVE/DEAD Far Red viability for 20 minutes 
625 at RT and then with anti-CD32 (FITC, Biolegend) and anti-CD3 (PerCP, Becton Dickinson) antibodies

626 for 20 minutes at RT. After the cell surface staining, cells were fixed and permeabilized with 627 Fixation/Permeabilization Solution (Becton Dickinson) for 20 mins at 4 으, washed with BD

628 Perm/Wash buffer, and stained with anti-p24-PE (Beckman Coulter) for 20 mins on ice and 20 mins 629 at RT. Finally, cells were washed and fixed with PFA (2\%). Samples were acquired in a FACSCalibur 630 flow cytometer (Becton Dickinson) and analyzed with FlowJo V10 software. The limit of detection 631 of the assay was established at 50 p24 $4^{+}$cells/million CD4+ $\mathrm{T}$ cells (Figure S3C).

\section{Quantification of HIV DNA and HIV RNA by quantitative PCR}

$\mathrm{CD}^{+} \mathrm{T}$ lymphocytes were enriched from total PBMCs using a negative selection kit (MagniSort 634 Human $\mathrm{CD}^{+}{ }^{+} \mathrm{T}$ Cell Enrichment, eBioscience). Then, $\mathrm{CD} 4^{+} \mathrm{T}$ cells were fractioned to extract RNA or 635 quantify the HIV DNA from cell lysates. CD4 ${ }^{+} \mathrm{T}$ cells for DNA analysis were immediately lysed with 636 proteinase $\mathrm{K}$-containing lysis buffer (at 55ㅇ $\mathrm{C}$ overnight and 95ㅇ $\mathrm{C}$ for 5 minutes). The HIV DNA in the 637 cell lysates was quantified by qPCR using primers and probes specific for the 1-LTR HIV region (LTR 638 forward 5'-TTAAGCCTCAATAAAGCTTGCC-3', LTR reverse 5'-GTTCGGGCGCCACTGCTAG-3' and probe 639 5' /56-FAM/CCAGAGTCA/ZEN/CACAACAGACGGGCA/31ABkFO/ 3'). CCR5 gene was used for cell 640 input normalization. Samples were analyzed in an Applied Biosystems 7000 Real-Time PCR System. 641 For Viral RNA quantification, $\mathrm{CD}^{+} \mathrm{T}$ cells were subjected to RNA extraction using the mirVana kit 642 following the manufacturer's instructions (Ambion). Reverse transcription of RNA to cDNA was

643 performed with SuperScriptIII (Invitrogen), and CDNA was quantified by qPCR with primers against 644 the HIV long terminal repeat (LTR). Quantification of RNA and DNA copies was performed using a 645 standard curve, and values were normalized to 1 million $\mathrm{CD}^{+} \mathrm{T}$ cells. was considered significant.

\section{Author contributions}

M.B. designed, directed, and interpreted experiments. A.A-G and J.G-E designed and performed 651 experiments, analyzed the data, and interpreted experiments. A.A-G, J.B, J.N, A.C, B.P, P.S, M.G, and 652 V.F were responsible for recruitment, specimen handling and storage, and related clinical data 
653 collection. A.A-G and M.B wrote the initial manuscript, and all of us contributed to the editing of the

654 manuscript.

\section{Acknowledgments}

This study was supported by the Spanish Secretariat of Science and Innovation and FEDER funds (grants SAF2015-67334-R and RTI2018-101082-B-I00 [MINECO/FEDER]), the Spanish “Ministerio de

658 Economia y Competitividad, Instituto de Salud Carlos III" (ISCIII, PI17/01470), GeSIDA and the

659 Spanish AIDS network Red Temática Cooperativa de Investigación en SIDA (RD16/0025/0007), the 660 Fundació La Marató TV3 (grants 201805-10FMTV3 and 201814-10FMTV3) and the Gilead 661 fellowships GLD19/00084 and GLD18/00008. M.B is supported by the Miguel Servet program 662 funded by the Spanish Health Institute Carlos III (CP17/00179). A.A-G is supported by the Spanish 663 Secretariat of Science and Innovation Ph.D. fellowship (BES-2016-076382). The funders had no role 664 in study design, data collection, and analysis, the decision to publish, or preparation of the 665 manuscript.

The authors declare that the data supporting the findings of this study are available within the paper and its supplementary information files. Source data are provided with this paper.

\section{REFERENCES}

671 1. Klatt, N.R., et al., Immune activation and HIV persistence: implications for curative approaches to HIV infection. Immunol Rev, 2013. 254(1): p. 326-42. 
686 8. Neidleman, J., et al., Phenotypic analysis of the unstimulated in vivo HIV CD4 T cell reservoir.

687

Elife, 2020. 9.

9. Descours, B., et al., CD32a is a marker of a CD4 T-cell HIV reservoir harbouring replicationcompetent proviruses. Nature, 2017. 543(7646): p. 564-567.

10. Bertagnolli, L.N., et al., The role of CD32 during HIV-1 infection. Nature, 2018. 561(7723): p. E17-E19.

11. Perez, L., et al., Conflicting evidence for HIV enrichment in CD32(+) CD4 T cells. Nature, 2018. 561(7723): p. E9-E16.

12. Darcis, G., et al., CD32(+)CD4(+) T Cells Are Highly Enriched for HIV DNA and Can Support Transcriptional Latency. Cell Rep, 2020. 30(7): p. 2284-2296 e3.

13. Abdel-Mohsen, M., et al., CD32 is expressed on cells with transcriptionally active HIV but does not enrich for HIV DNA in resting T cells. Sci Transl Med, 2018. 10(437).

14. Badia, R., et al., CD32 expression is associated to T-cell activation and is not a marker of the HIV-1 reservoir. Nat Commun, 2018. 9(1): p. 2739.

15. Vasquez, J.J., et al., CD32-RNA Co-localizes with HIV-RNA in CD3+ Cells Found within Gut Tissues from Viremic and ART-Suppressed Individuals. Pathog Immun, 2019. 4(1): p. 147160.

16. Noto, A., et al., CD32(+) and PD-1(+) Lymph Node CD4 T Cells Support Persistent HIV-1 Transcription in Treated Aviremic Individuals. J Virol, 2018. 92(20).

17. Martin, G.E., et al., CD32-Expressing CD4 T Cells Are Phenotypically Diverse and Can Contain Proviral HIV DNA. Front Immunol, 2018. 9: p. 928.

18. Gálvez, C., et al., Atlas of the HIV-1 Reservoir in Peripheral CD4 T Cells of Individuals on Successful Antiretroviral Therapy. 2021. 12(6): p. e0307821.

19. Serra-Peinado, C., et al., Expression of CD2O after viral reactivation renders HIV-reservoir cells susceptible to Rituximab. Nat Commun, 2019. 10(1): p. 3705.

20. Hogan, L.E., et al., Increased HIV-1 transcriptional activity and infectious burden in peripheral blood and gut-associated CD4+ $T$ cells expressing CD30. PLoS Pathog, 2018. 14(2): p. e1006856.

21. Vivier, E., et al., Functions of natural killer cells. Nat Immunol, 2008. 9(5): p. 503-10.

22. Lanier, L.L., Up on the tightrope: natural killer cell activation and inhibition. Nat Immunol, 2008. 9(5): p. 495-502.

23. Bottino, C., et al., Cellular ligands of activating NK receptors. Trends Immunol, 2005. 26(4): p. 221-6.

24. Andre, P., et al., Anti-NKG2A mAb Is a Checkpoint Inhibitor that Promotes Anti-tumor Immunity by Unleashing Both T and NK Cells. Cell, 2018. 175(7): p. 1731-1743 e13.

25. Pereira, B.I., et al., Senescent cells evade immune clearance via HLA-E-mediated NK and CD8(+) T cell inhibition. Nat Commun, 2019. 10(1): p. 2387.

26. Nabatanzi, R., et al., Aberrant natural killer (NK) cell activation and dysfunction among ARTtreated HIV-infected adults in an African cohort. Clin Immunol, 2019. 201: p. 55-60.

27. Lichtfuss, G.F., et al., Virologically suppressed HIV patients show activation of NK cells and persistent innate immune activation. J Immunol, 2012. 189(3): p. 1491-9.

28. Florez-Alvarez, L., J.C. Hernandez, and W. Zapata, NK Cells in HIV-1 Infection: From Basic Science to Vaccine Strategies. Front Immunol, 2018. 9: p. 2290.

29. Marras, F., et al., Control of the HIV-1 DNA Reservoir Is Associated In Vivo and In Vitro with NKp46/NKp30 (CD335 CD337) Inducibility and Interferon Gamma Production by Transcriptionally Unique NK Cells. J Virol, 2017. 91(23). 
752

753

754

755

756

757

758

759

760

761

762

763

764

765

766

767

768

769

770

771

772

773

774

775

776

777

30. Olesen, R., et al., Innate Immune Activity Correlates with CD4 T Cell-Associated HIV-1 DNA Decline during Latency-Reversing Treatment with Panobinostat. J Virol, 2015. 89(20): p. 10176-89.

31. Jost, S. and M. Altfeld, Evasion from NK cell-mediated immune responses by HIV-1. Microbes Infect, 2012. 14(11): p. 904-15.

32. Alpert, M.D., et al., ADCC develops over time during persistent infection with live-attenuated SIV and is associated with complete protection against SIV(mac)251 challenge. PLoS Pathog, 2012. 8(8): p. e1002890.

33. Pollara, J., et al., High-throughput quantitative analysis of HIV-1 and SIV-specific ADCCmediating antibody responses. Cytometry A, 2011. 79(8): p. 603-12.

34. Haynes, B.F., et al., Immune-correlates analysis of an HIV-1 vaccine efficacy trial. N Engl J Med, 2012. 366(14): p. 1275-86.

35. Gomez-Roman, V.R., et al., A simplified method for the rapid fluorometric assessment of antibody-dependent cell-mediated cytotoxicity. J Immunol Methods, 2006. 308(1-2): p. 5367.

36. Holgado, M.P., et al., CD32 Ligation Promotes the Activation of CD4(+) T Cells. Front Immunol, 2018. 9: p. 2814.

37. Garcia, M., et al., CD32 Expression is not Associated to HIV-DNA content in CD4 cell subsets of individuals with Different Levels of HIV Control. Sci Rep, 2018. 8(1): p. 15541.

38. Mikulak, J., et al., Natural killer cells in HIV-1 infection and therapy. AIDS, 2017. 31(17): p. 2317-2330.

39. Grau-Expósito J, S.-P.C., Miguel L, Navarro J, Curran A, Burgos J, Ocaña I, Ribera E, Torrella A, Planas B, Rosa Badía, Castellví J, Falcò V, Crespo M, Buzon MJ, A Novel Single-Cell FISHFlow Assay Identifies Effector Memory CD4 T cells as a Major Niche for HIV-1 Transcription in HIV-Infected Patients. mBIO, 2017.

40. Grau-Exposito, J., et al., Latency reversal agents affect differently the latent reservoir present in distinct CD4+ T subpopulations. PLoS Pathog, 2019. 15(8): p. e1007991.

41. Madhavi, V., et al., Antibody-dependent effector functions against HIV decline in subjects receiving antiretroviral therapy. J Infect Dis, 2015. 211(4): p. 529-38.

42. Tremblay-McLean, A., et al., Expression Profiles of Ligands for Activating Natural Killer Cell Receptors on HIV Infected and Uninfected CD4(+) T Cells. Viruses, 2017. 9(10).

43. Apps, R., et al., HIV-1 Vpu Mediates HLA-C Downregulation. Cell Host Microbe, 2016. 19(5): p. 686-95.

44. Ward, J.P., M.I. Bonaparte, and E. Barker, HLA-C and HLA-E reduce antibody-dependent natural killer cell-mediated cytotoxicity of HIV-infected primary T cell blasts. AIDS, 2004. 18(13): p. 1769-79.

45. Alevy, Y.G., J. Tucker, and T. Mohanakumar, CD32A (Fc gamma R/la) mRNA expression and regulation in blood monocytes and cell lines. Mol Immunol, 1992. 29(11): p. 1289-97.

46. Veri, M.C., et al., Monoclonal antibodies capable of discriminating the human inhibitory Fcgamma-receptor IIB (CD32B) from the activating Fcgamma-receptor IIA (CD32A): biochemical, biological and functional characterization. Immunology, 2007. 121(3): p. 392404.

47. Anania, J.C., et al., The Human FcgammaRII (CD32) Family of Leukocyte FcR in Health and Disease. Front Immunol, 2019. 10: p. 464.

48. Lu, J., et al., Structural recognition and functional activation of FcgammaR by innate pentraxins. Nature, 2008. 456(7224): p. 989-92. 
49. Clayton, K.L., et al., Resistance of HIV-infected macrophages to CD8(+) T lymphocytemediated killing drives activation of the immune system. Nat Immunol, 2018. 19(5): p. 475486.

50. Garrido, C., et al., Interleukin-15-Stimulated Natural Killer Cells Clear HIV-1-Infected Cells following Latency Reversal Ex Vivo. J Virol, 2018. 92(12).

51. Fromentin, R., et al., CD4+ T Cells Expressing PD-1, TIGIT and LAG-3 Contribute to HIV Persistence during ART. PLoS Pathog, 2016. 12(7): p. e1005761.

52. Huot, N., et al., CD32(+)CD4(+) $T$ Cells Sharing B Cell Properties Increase With Simian Immunodeficiency Virus Replication in Lymphoid Tissues. Front Immunol, 2021. 12: p. 695148.

53. Engelhardt, W., J. Matzke, and R.E. Schmidt, Activation-dependent expression of low affinity IgG receptors FC gamma RII(CD32) and Fc gamma RIII(CD16) in subpopulations of human $T$ lymphocytes. Immunobiology, 1995. 192(5): p. 297-320.

54. Gantner, P., et al., Single-cell TCR sequencing reveals phenotypically diverse clonally expanded cells harboring inducible HIV proviruses during ART. Nat Commun, 2020. 11(1): p. 4089.

55. Simonetti, F.R., et al., Clonally expanded CD4+ T cells can produce infectious HIV-1 in vivo. Proc Natl Acad Sci U S A, 2016. 113(7): p. 1883-8.

56. Alter, G., et al., HIV-1 adaptation to NK-cell-mediated immune pressure. Nature, 2011. 476(7358): p. 96-100.

57. Cohen, G.B., et al., The selective downregulation of class I major histocompatibility complex proteins by HIV-1 protects HIV-infected cells from NK cells. Immunity, 1999. 10(6): p. 66171.

58. Bonaparte, M.I. and E. Barker, Killing of human immunodeficiency virus-infected primary Tcell blasts by autologous natural killer cells is dependent on the ability of the virus to alter the expression of major histocompatibility complex class I molecules. Blood, 2004. 104(7): p. 2087-94.

59. Ren, Y., et al., BCL-2 antagonism sensitizes cytotoxic $T$ cell-resistant HIV reservoirs to elimination ex vivo. J Clin Invest, 2020. 130(5): p. 2542-2559.

60. Huang, S.H., et al., Latent HIV reservoirs exhibit inherent resistance to elimination by $C D 8+T$ cells. J Clin Invest, 2018. 128(2): p. 876-889.

61. Forthal, D.N. and A. Finzi, Antibody-dependent cellular cytotoxicity in HIV infection. AIDS (London, England), 2018. 32(17): p. 2439-2451.

62. Arias, J.F., et al., Tetherin antagonism by Vpu protects HIV-infected cells from antibodydependent cell-mediated cytotoxicity. Proc Natl Acad Sci U S A, 2014. 111(17): p. 6425-30.

63. Veillette, M., et al., Interaction with cellular CD4 exposes HIV-1 envelope epitopes targeted by antibody-dependent cell-mediated cytotoxicity. J Virol, 2014. 88(5): p. 2633-44.

64. Murin, C.D., Considerations of Antibody Geometric Constraints on NK Cell Antibody Dependent Cellular Cytotoxicity. Front Immunol, 2020. 11: p. 1635.

65. Martina, B.E., P. Koraka, and A.D. Osterhaus, Dengue virus pathogenesis: an integrated view. Clin Microbiol Rev, 2009. 22(4): p. 564-81.

66. Lu, L.L., et al., Beyond binding: antibody effector functions in infectious diseases. Nat Rev Immunol, 2018. 18(1): p. 46-61.

67. Bournazos, S. and J.V. Ravetch, Fcgamma Receptor Function and the Design of Vaccination Strategies. Immunity, 2017. 47(2): p. 224-233.

68. Hessell, A.J., et al., Fc receptor but not complement binding is important in antibody protection against HIV. Nature, 2007. 449(7158): p. 101-4. 
825

826

827

828

829

830

831

832

833

834

835

836

837

838

839

840

841

842

843

844

845

846

847

848

849

850

851

852

853

854

855

856

857

858

859

860

69. Bournazos, S., et al., Broadly neutralizing anti-HIV-1 antibodies require Fc effector functions for in vivo activity. Cell, 2014. 158(6): p. 1243-1253.

70. Martini, F., et al., HLA-E up-regulation induced by HIV infection may directly contribute to CD94-mediated impairment of NK cells. Int J Immunopathol Pharmacol, 2005. 18(2): p. 26976.

71. Kanevskiy, L., et al., Dimorphism of HLA-E and its Disease Association. Int J Mol Sci, 2019. 20(21).

72. Kaiser, B.K., et al., Structural basis for NKG2A/CD94 recognition of HLA-E. Proc Natl Acad Sci U S A, 2008. 105(18): p. 6696-701.

73. Brostjan, C., et al., Differential expression of inhibitory and activating CD94/NKG2 receptors on NK cell clones. J Immunol Methods, 2002. 264(1-2): p. 109-19.

74. Ward, A.R., T.M. Mota, and R.B. Jones, Immunological approaches to HIV cure. Semin Immunol, 2020: p. 101412.

75. Spivak, A.M. and V. Planelles, Novel Latency Reversal Agents for HIV-1 Cure. Annu Rev Med, 2018. 69: p. 421-436.

76. Deng, K., et al., Broad CTL response is required to clear latent HIV-1 due to dominance of escape mutations. Nature, 2015. 517(7534): p. 381-5.

77. Merino, A., et al., Chronic stimulation drives human NK cell dysfunction and epigenetic reprograming. J Clin Invest, 2019. 129(9): p. 3770-3785.

78. Mori, S., et al., Differential regulation of human NK cell-associated gene expression following activation by IL-2, IFN-alpha and PMA/ionomycin. Int J Oncol, 1998. 12(5): p. 1165-70.

79. Jensen, S.S., et al., HIV-Specific Antibody-Dependent Cellular Cytotoxicity (ADCC) -Mediating Antibodies Decline while NK Cell Function Increases during Antiretroviral Therapy (ART). PLoS One, 2015. 10(12): p. e0145249.

80. Sengupta, S. and R.F. Siliciano, Targeting the Latent Reservoir for HIV-1. Immunity, 2018. 48(5): p. 872-895.

81. Halvas, E.K., et al., HIV-1 viremia not suppressible by antiretroviral therapy can originate from large T cell clones producing infectious virus. J Clin Invest, 2020. 130(11): p. 5847-5857.

82. Li, M., et al., Genetic and neutralization properties of subtype C human immunodeficiency virus type 1 molecular env clones from acute and early heterosexually acquired infections in Southern Africa. J Virol, 2006. 80(23): p. 11776-90.

83. Moore, J.P., et al., Immunochemical analysis of the gp120 surface glycoprotein of human immunodeficiency virus type 1: probing the structure of the C4 and V4 domains and the interaction of the C4 domain with the V3 loop. J Virol, 1993. 67(8): p. 4785-96. 
bioRxiv preprint doi: https://doi.org/10.1101/2022.02.24.481766; this version posted February 25, 2022. The copyright holder for this preprint (which was not certified by peer review) is the author/funder. All rights reserved. No reuse allowed without permission.

Table S1. Clinical data of PLWH included in the study.

\begin{tabular}{|c|c|c|c|c|c|c|}
\hline $\begin{array}{l}\# \\
\text { Patient } \\
\text { ID }\end{array}$ & $\begin{array}{l}\text { Time since HIV } \\
\text { diagnosis } \\
\text { (months) }\end{array}$ & $\begin{array}{l}\text { CD4 Cell } \\
\text { Count } \\
\text { (cells } / \mu \mathrm{l})\end{array}$ & \%CD4 & $\begin{array}{l}\text { Viral Load } \\
\text { (copies/ml) }\end{array}$ & $\begin{array}{l}\text { Time on ART- VL- } \\
\text { suppressed } \\
\text { (months) }\end{array}$ & ART regimen \\
\hline 1 (EC) & 287 & 1170 & 52.8 & $<50$ & - & UNT \\
\hline 2 (EC) & 11 & 840 & 23.7 & $<50$ & - & UNT \\
\hline 3 (EC) & 51 & 1030 & 36.0 & $<50$ & - & UNT \\
\hline $4(E C)$ & 104 & 1610 & 46.0 & $<50$ & - & UNT \\
\hline $5(E C)$ & 59 & 890 & NA & $<50$ & - & UNT \\
\hline 6 (EC) & 321 & 490 & 20.4 & $<50$ & - & UNT \\
\hline 7 (EC) & 189 & 410 & 23.2 & $<50$ & - & UNT \\
\hline 8 & 221 & 1240 & 40.1 & $<50$ & 158 & $E F V+T D F+3 T C$ \\
\hline 9 & 373 & 960 & 33.7 & $<50$ & 113 & $C O B+D R V$ \\
\hline 10 & 301 & 1050 & 27.0 & $<50$ & 142 & $\mathrm{COB}+\mathrm{DRV}$ \\
\hline 11 & 102 & 1040 & 37.0 & $<50$ & 61 & $\mathrm{DRV}+\mathrm{RTV}+3 T \mathrm{C}$ \\
\hline 12 & 128 & 760 & 28.1 & $<50$ & 59 & $3 T C+A B V+R P V$ \\
\hline 13 & 73 & 660 & 40.4 & $<50$ & 67 & $3 T C+A B V+N V P$ \\
\hline 14 & 333 & 670 & 24.1 & $<50$ & 86 & $\mathrm{RPV}+\mathrm{COB}+\mathrm{DRV}$ \\
\hline 15 & 67 & 830 & 28.6 & $<50$ & 62 & $3 T C+A B V+R P V$ \\
\hline 16 & 142 & 630 & 36.0 & $<50$ & 85 & $\mathrm{FTC}+\mathrm{RPV}+\mathrm{TDF}$ \\
\hline 17 & 213 & 1000 & 42.4 & $<50$ & 76 & $\mathrm{FTC}+\mathrm{RPV}+\mathrm{TDF}$ \\
\hline 18 & 107 & 600 & 31.0 & $<50$ & 105 & $\mathrm{FTC}+\mathrm{RPV}+\mathrm{TDF}$ \\
\hline 19 & 161 & 1150 & 36.5 & $<50$ & 85 & $\mathrm{FTC}+\mathrm{NVP}+\mathrm{TDF}$ \\
\hline 20 & 90 & 710 & 38.3 & $<50$ & 85 & $\mathrm{FTC}+\mathrm{TDF}+\mathrm{COB}+\mathrm{ATV}$ \\
\hline 21 & 136 & 810 & 44.3 & $<50$ & 90 & $3 T C+A B V+E F V$ \\
\hline 22 & 86 & 1000 & 37.0 & $<50$ & 66 & $\mathrm{FTC}+\mathrm{RPV}+\mathrm{TDF}$ \\
\hline 23 & 319 & 689 & 24.5 & $<50$ & 115 & $E T V+R T G+R T V+D R V$ \\
\hline 24 & 54 & 530 & 24.0 & $<50$ & 51 & $R T G+3 T C+A B V$ \\
\hline 25 & 119 & 750 & 27.9 & $<50$ & 97 & $3 T C+A B V+D T G$ \\
\hline 26 & 95 & 660 & 49.5 & $<50$ & 69 & $\mathrm{ETV}+\mathrm{FTC}++\mathrm{TDF}$ \\
\hline 27 & 323 & 2090 & 46.0 & $<50$ & 43 & $3 T C+A B V+D T G$ \\
\hline 28 & 240 & NA & 25.6 & $<50$ & 71 & TDF+COB+DRV \\
\hline 29 & 76 & 540 & 25.0 & $<50$ & 56 & $\mathrm{RPV}+\mathrm{FTC}+\mathrm{TDF}$ \\
\hline 30 & 65 & 570 & 26.2 & $<50$ & 62 & $3 T C+A B V+R T G$ \\
\hline 31 & 248 & 1050 & 32.0 & $<50$ & 84 & $\mathrm{COB}+\mathrm{DRV}$ \\
\hline 32 & 293 & 560 & 24.4 & $<50$ & 80 & $\mathrm{RTG}+\mathrm{COB}+\mathrm{DRV}$ \\
\hline 33 & 113 & 600 & 38.7 & $<50$ & 95 & $\mathrm{EFV}+\mathrm{FTC}+\mathrm{TDF}$ \\
\hline 34 & 267 & 570 & 18.1 & $<50$ & 98 & $\mathrm{RTG}+\mathrm{FTC}+\mathrm{TAF}$ \\
\hline 35 & 134 & 870 & 48.7 & $<50$ & 97 & $C O B+D R V+D T G$ \\
\hline 36 & 98 & 680 & 54.0 & $<50$ & 86 & $3 T C+A B V+R P V$ \\
\hline 37 & 54 & 350 & 24.0 & $<50$ & 48 & $\mathrm{RTV}+\mathrm{FTC}+\mathrm{TDF}$ \\
\hline 38 & 121 & 850 & 35.6 & $<50$ & 88 & $3 T C+A B V+D T G$ \\
\hline 39 & 286 & 1060 & 30.3 & $<50$ & 82 & $3 T C+A B V+D T G$ \\
\hline 40 & 99 & 930 & 46.0 & $<50$ & 79 & $\mathrm{RPV}+\mathrm{FTC}+\mathrm{TDF}$ \\
\hline
\end{tabular}


bioRxiv preprint doi: https://doi.org/10.1101/2022.02.24.481766; this version posted February 25, 2022. The copyright holder for this preprint

\begin{tabular}{|c|c|c|c|c|c|c|}
\hline 41 & 94 & 1160 & 31.0 & $<50$ & 73 & $3 T C+A B V+D T G$ \\
\hline 42 & 73 & 310 & 13.3 & $<50$ & 67 & $3 T C+A B V+R P V$ \\
\hline 43 & 99 & 450 & 25.0 & $<50$ & 96 & $3 T C+A B V+R P V$ \\
\hline 44 & 89 & 690 & 35.2 & $<50$ & 85 & $\mathrm{RPV}+\mathrm{FTC}+\mathrm{TDF}$ \\
\hline 45 & 98 & 800 & 40.2 & $<50$ & 89 & $\mathrm{FTC}+\mathrm{RTV}+\mathrm{TAF}$ \\
\hline 46 & 63 & 660 & 29.8 & $<50$ & 24 & $\mathrm{BIC}+\mathrm{FTC}+\mathrm{TAF}$ \\
\hline 47 & 116 & 550 & 38.4 & $<50$ & 92 & $\mathrm{FTC}+\mathrm{RPV}+\mathrm{TDF}$ \\
\hline 48 & 169 & 1040 & 37.4 & $<50$ & 101 & $3 T C+A B V+N V P$ \\
\hline 49 & 146 & 860 & 37.4 & $<50$ & 54 & $\mathrm{RPV}+\mathrm{FTC}+\mathrm{TDF}$ \\
\hline 50 & 60 & 890 & 34.5 & $<50$ & 55 & $3 T C+A B V+D T G$ \\
\hline 51 & 40 & 570 & 27.4 & $<50$ & 28 & $3 T C+A B V+D T G$ \\
\hline 52 & 214 & 890 & 37.0 & $<50$ & 99 & $\mathrm{EFV}+\mathrm{FTC}+\mathrm{TDF}$ \\
\hline 53 & 135 & 790 & 27.5 & $<50$ & 90 & $\mathrm{RPV}+\mathrm{FTC}+\mathrm{TDF}$ \\
\hline 54 & 90 & 640 & 24.2 & $<50$ & 62 & $\mathrm{DRV}+\mathrm{FTC}+\mathrm{TDF}+\mathrm{RTV}$ \\
\hline 55 & 193 & 890 & 34.1 & $<50$ & 85 & $\mathrm{EFV}+\mathrm{FTC}+\mathrm{TDF}$ \\
\hline 56 & 80 & 500 & 28.6 & $<50$ & 72 & $\mathrm{RPV}+\mathrm{FTC}+\mathrm{TDF}$ \\
\hline 57 & 178 & 850 & 39.5 & $<50$ & 81 & $\mathrm{EFV}+\mathrm{FTC}+\mathrm{TDF}$ \\
\hline 58 & 103 & 1170 & 44.0 & $<50$ & 58 & $\mathrm{RPV}+\mathrm{FTC}+\mathrm{TDF}$ \\
\hline 59 & 72 & 800 & 23.1 & $<50$ & 18 & $\mathrm{ABV} / 3 \mathrm{TC}+\mathrm{DTG}$ \\
\hline 60 & 33 & 490 & 21.0 & $<50$ & 28 & TDF/FTC+EVG/C \\
\hline 61 & 31 & 560 & 27.9 & $<50$ & 11 & TDF/FTC+EVG/C \\
\hline 62 & 49 & 1070 & 38.1 & $<50$ & 39 & $\mathrm{TDF}+\mathrm{FTC}+\mathrm{EFV}$ \\
\hline 63 & 31 & 810 & 34.7 & $<50$ & 22 & $A B V / 3 T C+D T G$ \\
\hline 64 & 42 & 1150 & 37.0 & $<50$ & 37 & $\mathrm{ABV} / 3 \mathrm{TC}+\mathrm{RPV}$ \\
\hline 65 & 13 & 1760 & 57.7 & $<50$ & $>13$ & TDF/FTC+EVG/C \\
\hline 66 & 55 & 970 & NA & $<50$ & 45 & $\mathrm{ABV} / 3 \mathrm{TC}+\mathrm{DGT}$ \\
\hline 67 & 168 & 540 & NA & $<50$ & 54 & TDF/FTC+EVG/C \\
\hline 68 & 38 & 920 & 39.8 & $<50$ & 27 & $\mathrm{COB}+\mathrm{FTC}+\mathrm{TAF}+\mathrm{EVG}$ \\
\hline 69 & 83 & 730 & 34.2 & $<50$ & 25 & $\mathrm{COB}+\mathrm{FTC}+\mathrm{TAF}+\mathrm{EVG}$ \\
\hline 70 & 44 & 360 & 31.0 & $<50$ & 35 & $R T G+3 T C+A B V$ \\
\hline 71 & 116 & 590 & 23.0 & $<50$ & 96 & $\mathrm{RPV}+\mathrm{FTC}+\mathrm{TDF}$ \\
\hline 72 & 72 & 930 & 44.8 & 50 & 60 & 3TC+DTG \\
\hline 73 & 106 & 580 & 28.3 & $<50$ & 34 & $\mathrm{BIC}+\mathrm{FTC}+\mathrm{TAF}$ \\
\hline 74 & 97 & 640 & 29.0 & $<50$ & 63 & $\mathrm{FTC}+\mathrm{RPV}+\mathrm{TAF}$ \\
\hline 75 & 96 & 540 & 35.0 & 50 & 74 & $\mathrm{RPV}+\mathrm{FTC}+\mathrm{TDF}$ \\
\hline 76 & 121 & 810 & 38.4 & $<50$ & 45 & $\mathrm{BIC}+\mathrm{FTC}+\mathrm{TAF}$ \\
\hline 77 & 169 & 650 & 35.7 & $<50$ & 92 & $3 T C+A B V+D T G$ \\
\hline 78 & 163 & 600 & 29.3 & $<50$ & 70 & $\mathrm{FTC}+\mathrm{RPV}+\mathrm{TDF}$ \\
\hline 79 & 105 & 670 & 32.1 & $<50$ & 103 & $\mathrm{FTC}+\mathrm{RPV}+\mathrm{TDF}$ \\
\hline 80 & 156 & 700 & 48.5 & $<50$ & 118 & $\mathrm{COB}+\mathrm{DRV}+\mathrm{DTG}$ \\
\hline 81 & 62 & 1420 & 44.3 & $<50$ & 54 & $3 T C+D T G$ \\
\hline 82 & 319 & 280 & 27.0 & $<50$ & 70 & $M R V+D R V+R T V+D T G$ \\
\hline 83 & 100 & 1080 & 37.4 & $<50$ & 84 & $\mathrm{RPV}+\mathrm{FTC}+\mathrm{TDF}$ \\
\hline 84 & 51 & 860 & 31.2 & $<50$ & 33 & $3 T C+A B V+D T G$ \\
\hline 85 & 156 & 1240 & 44.0 & $<50$ & 35 & $\mathrm{BIC}+\mathrm{FTC}+\mathrm{TDF}$ \\
\hline 86 & 158 & 990 & 48.9 & $<50$ & 81 & $3 T C+A B V+D T G$ \\
\hline
\end{tabular}


bioRxiv preprint doi: https://doi.org/10.1101/2022.02.24.481766; this version posted February 25, 2022. The copyright holder for this preprint (which was not certified by peer review) is the author/funder. All rights reserved. No reuse allowed without permission.

$\begin{array}{lcccccc}87 & 58 & 600 & 32.1 & <50 & 56 & \text { DTG+3TC } \\ 88 & 448 & 1420 & 44.0 & <50 & 42 & \text { COB+DRV+RPV } \\ 89 & 31 & 480 & 31.2 & <50 & 28 & \text { DRV+COB+FTC+TAF }\end{array}$

EC, Elite Controller; FTC, emtricitabine; TDF, tenofovir; NVP, nevirapine; ATV, atazanavir; 3TC, lamivudine; EFV, efavirenz; ABV, abacavir; RAL, raltegravir; EVG, elvitegravir; DTG, dolutegravir; DRV, darunavir; RPV, Rilpivirine; TAF, tenofovir alafenamida; BIC, Bictegravir; LPV, Lopinavir; /r, boosted with ritonavir; /c, boosted with cobicistat; UNT, untreated; NA: Not Available. 
Figure 1

A

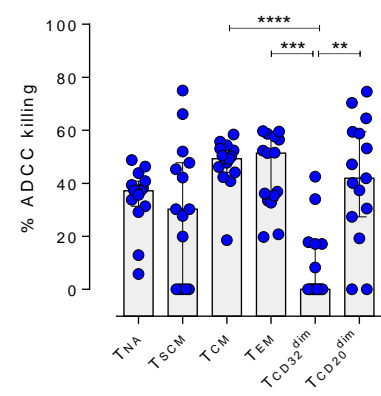

B

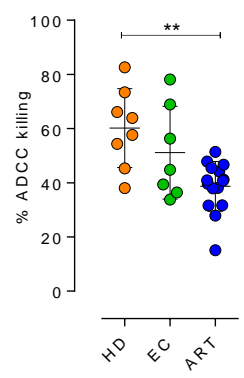

C

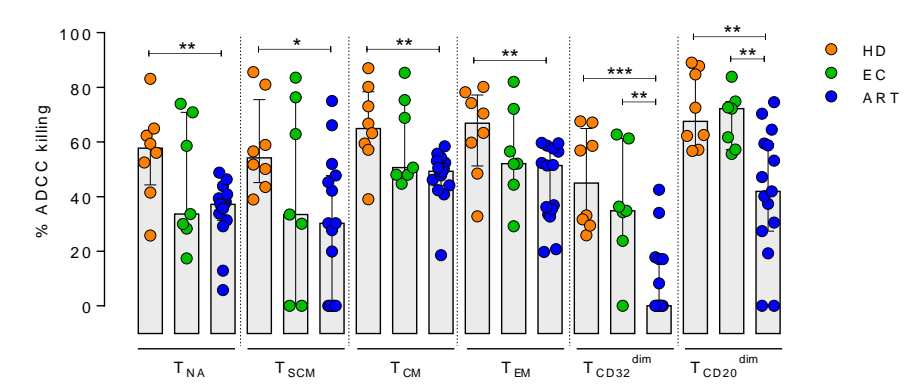

D

E

F
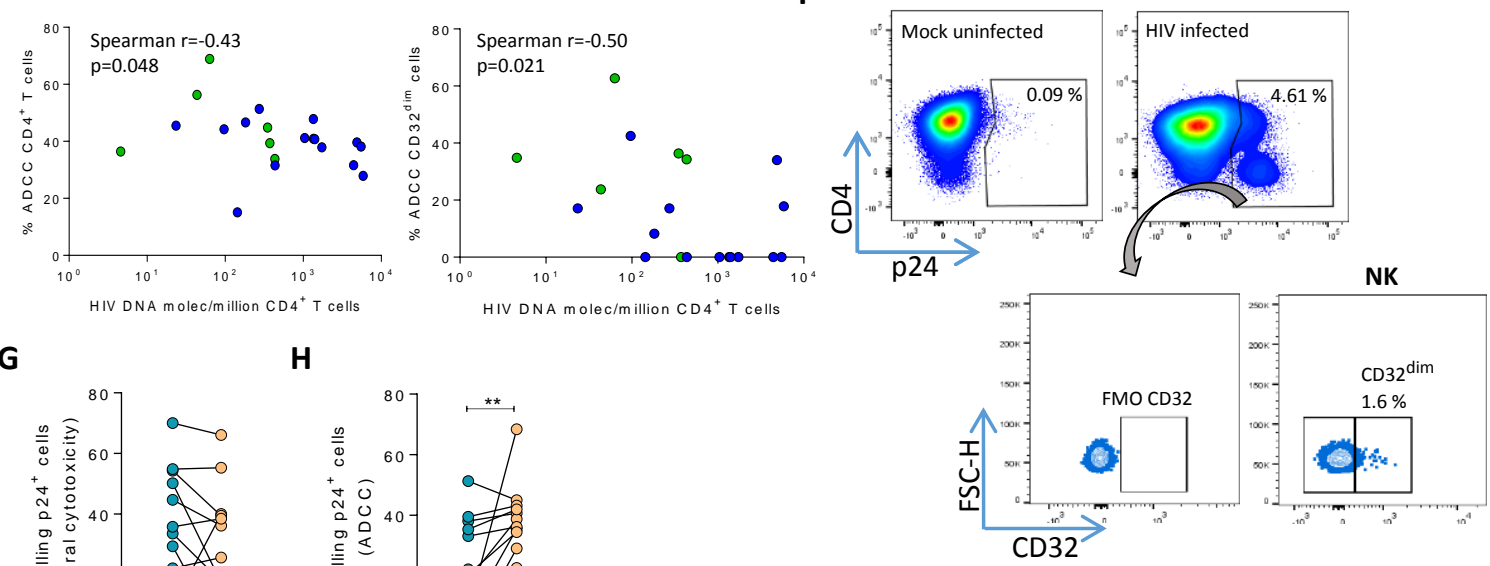

NK+Plasma HIV+

G

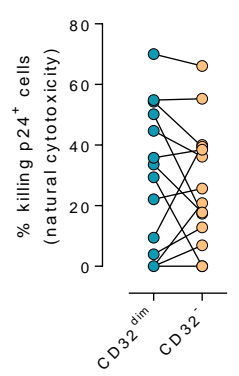

H
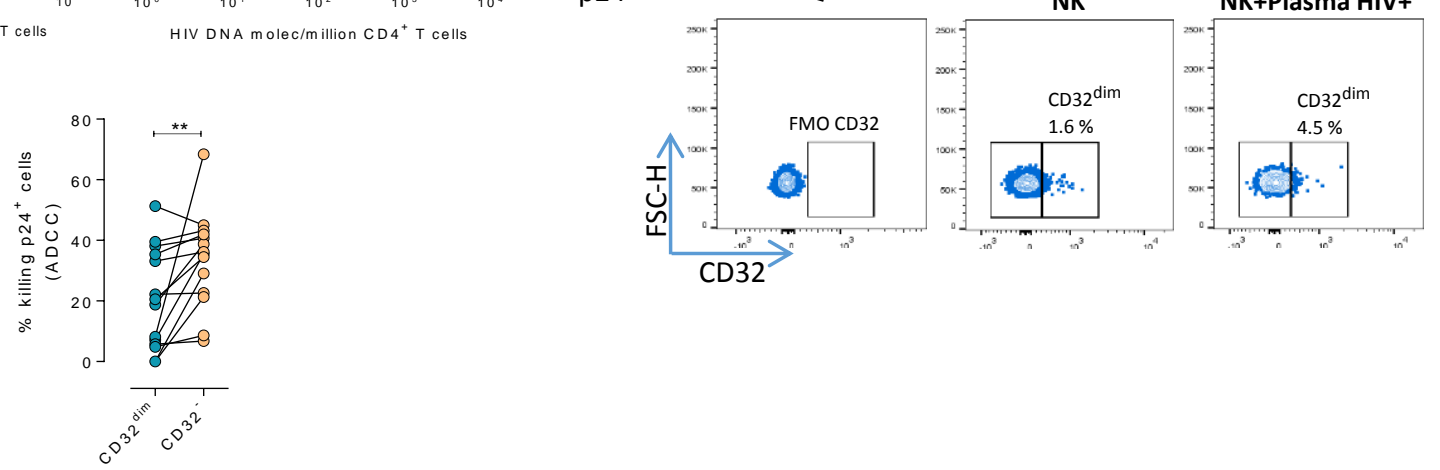

Figure 1. Susceptibility of $\mathrm{CD4}^{+} \mathrm{T}$ cell subsets to the NK immune response. The susceptibility of different cell subpopulations that compose the HIV-reservoir to Natural Cytotoxicity (NC) and Antibody-Dependent Cell Cytotoxicity (ADCC) mediated by NK cells was measured by performing functional assays. A) Percentage of gp120 coated cells killed by ADCC after being exposed to HIV-specific immunoglobulins (Igs) in the presence of NK cells. The intrinsic susceptibility to ADCC was measured in Naïve $\left(T_{N A}\right)$, Stem Cell Memory $\left(T_{S C M}\right)$, Central Memory $\left(T_{C M}\right)$, Effector Memory $\left(T_{E M}\right), T_{C D 32}{ }^{d i m}$, and $T_{C D 20}$ dim subsets. Statistical comparisons were performed using the ANOVA Friedman test. Median with interquartile range is shown. B) Percentage of total gp120 coated CD4 ${ }^{+} \mathrm{T}_{\text {cells from }}$ different cohorts of patients killed by ADCC. Healthy donors (HD), Elite Controllers (EC), and antiretroviral treated PLWH (ART). Statistical comparisons were performed using the Mann-Whitney test. Median with interquartile range are shown. C) Percentage of cell subsets killed by ADCC in cells from HD, EC, and ART. Statistical comparisons were performed using the Mann-Whitney test. Median with interquartile range is shown. D-E) Spearman correlations between the size of the HIV-reservoir measured as total HIV-DNA in samples from ART-suppressed PLWH, and the potency of autologous NK cells to kill (D) total CD4+ $T$ cells or (E) $T_{C D 32} \operatorname{dim}$ cells by $A D C C$. F) Representative flow cytometry gating strategy used to quantify HIV infection after ex vivo infection with BaL or NL4.3. Fluorescence minus one (FMO) control was used to determine CD32 expression. Cells were infected for 5 days and the frequency of expression of CD32 on HIV-infected cells was measured for each condition. G) Percentage of killing by NC of ex-vivo HIV-infected $\mathrm{T}_{\mathrm{CD} 32}$ dim and $\mathrm{T}_{\mathrm{CD} 32}$ - cells mediated by autologous NK cells from ART-treated PLWH $(n=14)$. Killing was calculated by normalizing the proportion of each subset within the p24 fraction in the co-culture condition to the basal condition. H) Percentage of ADCC killing of ex vivo HIV-infected $T_{C D 32}{ }^{\text {dim }}$ and $T_{C D 32}$ cells mediated by autologous NK cells from ART-treated PLWH $(n=14)$. Killing was calculated by normalizing the proportion of each subset within the $\mathrm{p} 24^{+}$fraction in the co-culture condition with plasma to the co-culture without plasma. Statistical comparisons were performed using the Wilcoxon matched-pairs signed rank test. ${ }^{*} p<0.05 ; * * p<0.01$. 


\section{Figure 2}

A

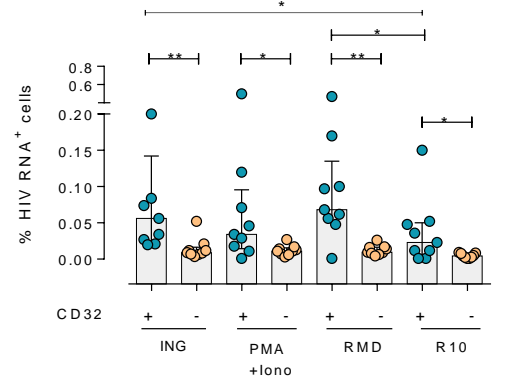

E

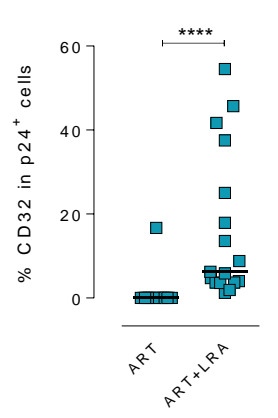

B

$\mathbf{F}$

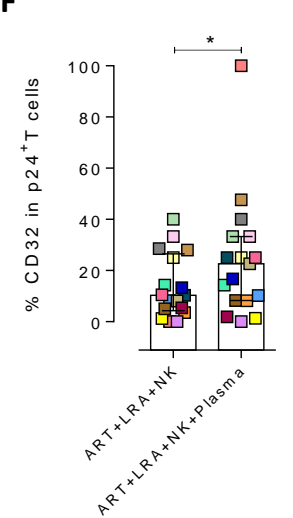

C

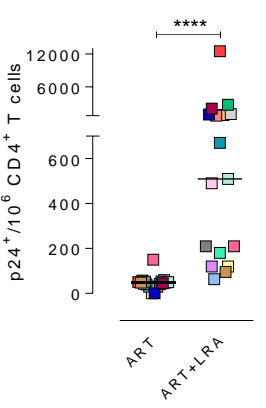

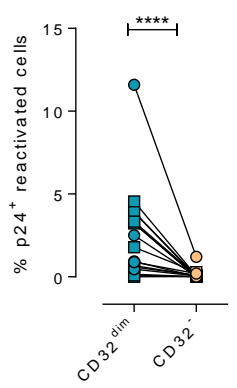

G
D

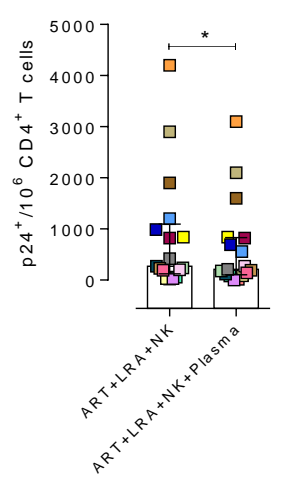

Figure 2. Expression of HIV in $\mathrm{T}_{\mathrm{CD} 32}$ dim reservoir cells after latency disruption and susceptibility to NK immune responses. Data from the direct ex vivo reactivation of the natural HIV reservoir in ART-suppressed PLWH. A) Percentage of HIV-RNA expressing cells, measured by the RNA FISH-flow assay, within the $T_{C D 32}{ }^{d i m}$ and $T_{C D 32}{ }^{-}$ subsets after viral reactivation with Ingenol, $\mathrm{PMA} /$ ionomycin, or romidepsin $(\mathrm{n}=9)$. Median with interquartile range is represented. B) Percentage of $\mathrm{p}^{+} 4^{+}$cells after $18 \mathrm{~h}$ viral reactivation with PMA/ionomycin $(n=17)$. Each participant is represented by a different color. C) Frequency of viral reactivation within the total pool of $T_{C D 32}$ dim and $\mathrm{T}_{\mathrm{CD} 32}{ }^{-}$cells $(\mathrm{n}=17)$. D) NK killing assays against viral reactivated cells. Number of $\mathrm{p} 24^{+}$cells per million $\mathrm{CD}^{+}{ }^{+} \mathrm{T}$ cells after the addition of NK cells only or together with the autologous plasma is shown $(n=17)$. E) Percentage of CD32 expression within the total p24+ pool before and after HIV reactivation $(n=17)$. F) Percentage of $T_{C D 32}$ dim within $\mathrm{p} 24^{+}$cells after HIV reactivation and functional NK-mediated assays $(n=17)$. G) Percentage of NK-mediated killing by ADCC of the reactivated $T_{C D 32}{ }^{\text {dim }}$ or total $p 24^{+}$cells $(n=17)$. ADCC was calculated as the reduction of $p 24^{+}$ cells after the co-culture with NK and plasma and normalized to the condition with NK cells alone. 
Figure 3

A

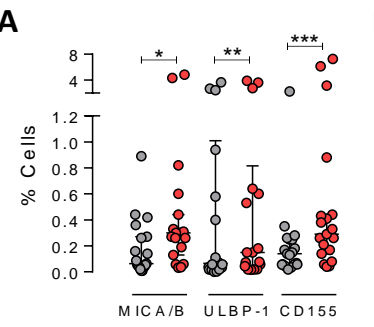

B

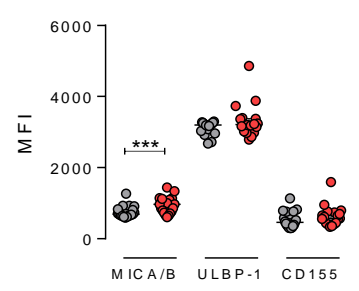

C

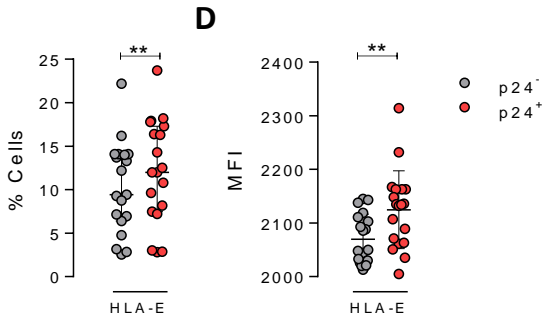

E

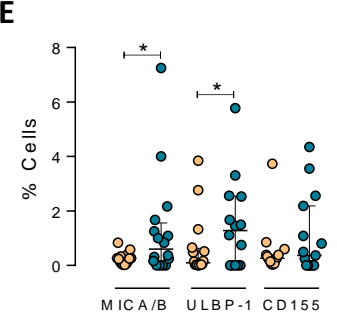

F

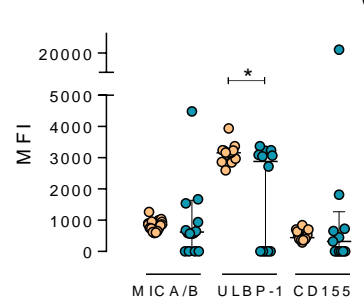

G

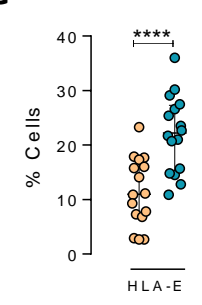

H

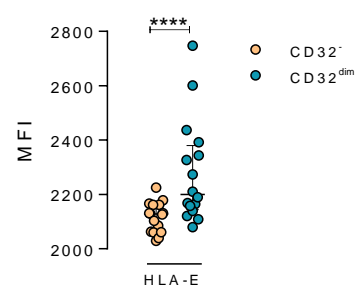

I

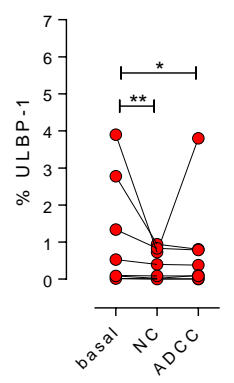

M

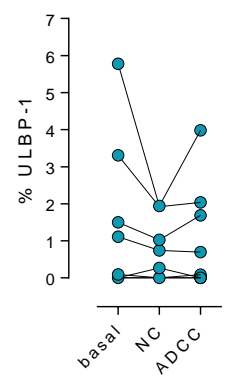

J

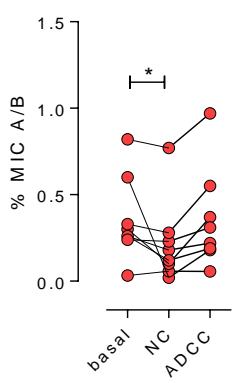

N

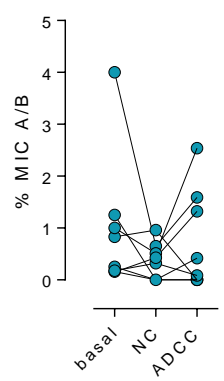

K

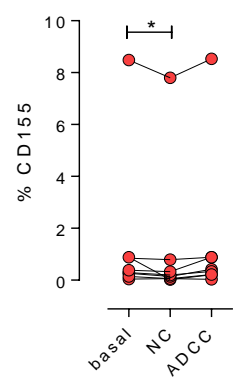

0

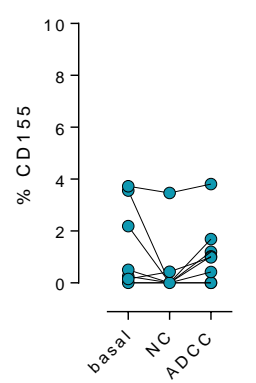

L

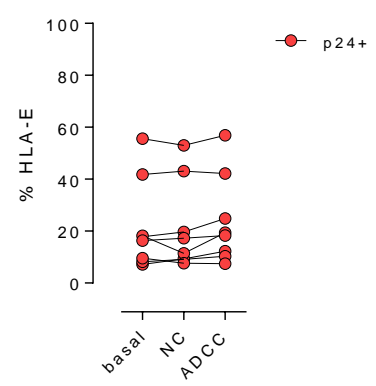

P

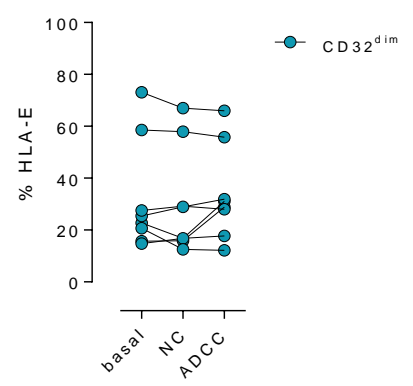

Figure 3. Expression of NK-ligands in cells resistant to NK-mediated killing. HIV-infected cells from healthy donors were subjected to NK-killing assays and the percentage of expression of different NK-ligands was measured by flow cytometry in different fractions (CD32 and CD32 $\left.{ }^{\mathrm{dim}}\right)$ of infected (p24+) or uninfected (p24-) cells. A-H) Expression of NK-ligands before performing the killing assays $(n=19)$ : A) Percentage of $C D 4^{+}$T cells expressing MIC A/B, ULBP-1, and CD155. B) Mean Fluorescence Intensity (MFI) values for the expression of MIC A/B, ULBP-1, and CD155 on CD4 T cells. C) Percentage of $\mathrm{CD}^{+}{ }^{+} \mathrm{T}$ cells expressing the MHC molecule HLA-E. D) MFI values for HLA-E expression on $\mathrm{CD}^{+} \mathrm{T}$ cells. E-H) Same analyses as A-D but showing HIV-infected CD32 ${ }^{\text {dim }}$ and CD32- cells. I-P) Expression of NKligands on HIV-infected cells not killed by the different NK-killing mechanisms. Natural Cytotoxicity (NC) and Antibody-Dependent Cell Cytotoxicity (ADCC). I-L) Expression of NK-ligands on total infected CD4 ${ }^{+}$cells before and after NK killing. M-P) Expression of NK-ligands on infected $T_{C D 32}$ dim cells before and after NK killing. All graphs show median with interquartile range and the statistical comparisons were performed using the Wilcoxon matched-pairs signed rank test. ${ }^{*} p<0.05 ; * * p<0.01 ; * * * p<0.001 ; * * * * p<0.0001$. 
Figure 4

A

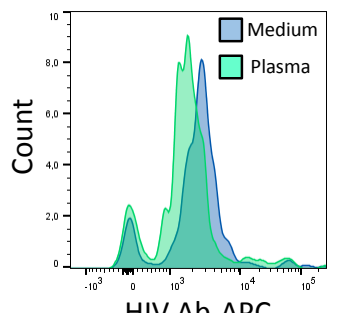

B

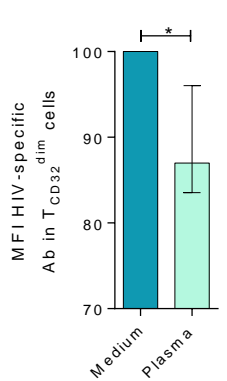

C

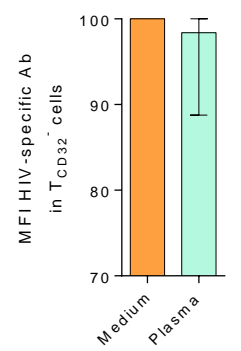

D

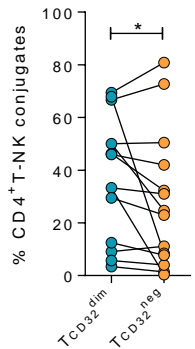

$\mathbf{E}$

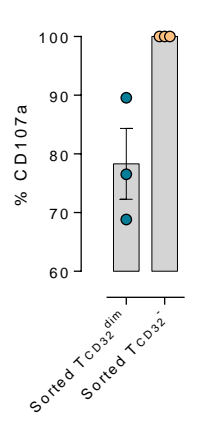

$\mathbf{F}$

F $\because$ FcgammaR CD32
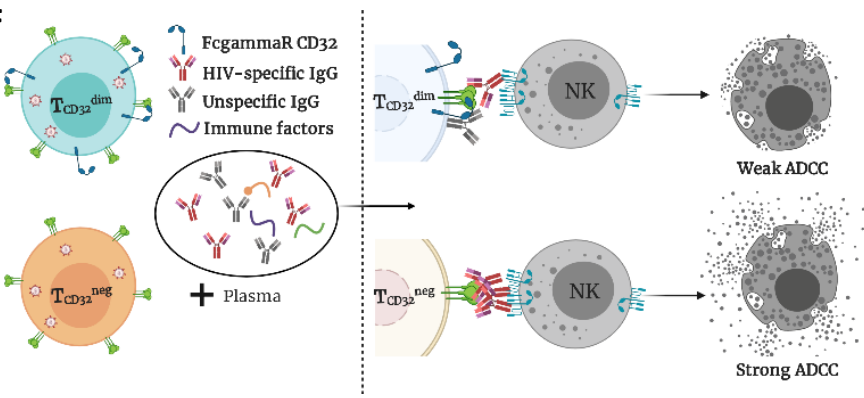

Figure 4. Reduced binding of HIV-specific antibodies to $\mathrm{T}_{\mathrm{CD} 32}{ }^{\text {dim }}$ cells and the effect on NK degranulation. The binding capability of the HIVgp120-specific IgG A32, an antibody (Ab) labelled with allophycocyanin (APC), to gp120-coated $\mathrm{T}_{\mathrm{CD} 32}$ dim and $\mathrm{T}_{\mathrm{CD} 32}{ }^{-}$cells from healthy donors, before and after incubation with plasma containing non-HIV specific IgGs, was measured by flow cytometry $(n=7)$. Percentage of the Mean Fluorescence Intensity (MFI) signal, normalized to the medium, for $\mathrm{A}^{2} 2^{+}$cells after plasma addition is shown in A) Representative histogram of $\mathrm{A32}^{+} \mathrm{T}_{\mathrm{CD} 32}{ }^{\operatorname{dim}}$ cells, B) $\mathrm{T}_{\mathrm{CD} 32}{ }^{\operatorname{dim}}$ and C) $\mathrm{T}_{\mathrm{CD} 32}$ cells. D) Percentage of cell conjugates between ex vivo HIV-infected $T_{C D 32}$ dim or $T_{C D 32}$ and NK cells after performing $A D C C$ assays $(n=14)$. E) Percentage of NK degranulation (CD107a marker) in cell conjugates with sorted $\mathrm{T}_{\mathrm{CD} 32}$ dim or $\mathrm{T}_{\mathrm{CD} 32}{ }^{-}$coated with gp120 HIV protein and incubated with plasma $\mathrm{HIV}^{+}$, after a $4 \mathrm{~h}$ ADCC activation assay $(\mathrm{n}=3)$. Values are normalized to the CD32population. F) Schematic illustration of the impaired ADCC response against $T_{C D 32}{ }^{\text {dim }}$ cells. Graphs show median with range and statistical comparisons were performed using Wilcoxon matched-pairs signed rank test. ${ }^{*} p<0.05$. 
Figure 5

A

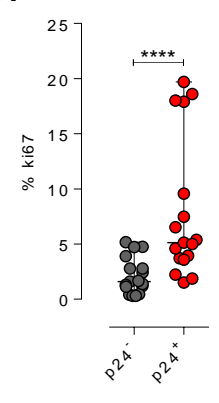

B

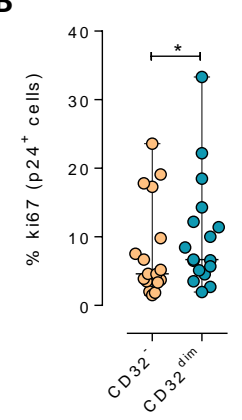

C

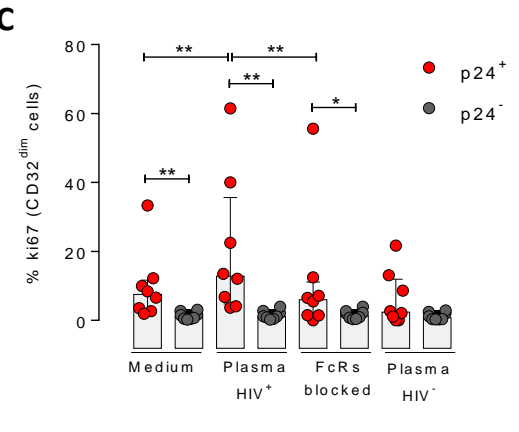

D

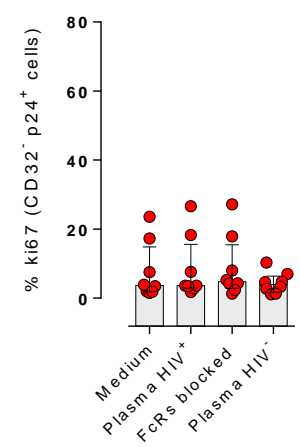

Figure 5. Immune complexes engagement with CD32 induces proliferation in HIV-infected CD32 ${ }^{\text {dim }}$ cells. Cells from healthy donors were infected with the viral strain $\mathrm{HIV}_{\mathrm{BaL}}$ and 5 days post-infection Ki67 expression was measured by flow cytometry. A) Expression of the proliferation marker Ki67 in uninfected or ex-vivo HIV-infected $\mathrm{CD4}^{+} \mathrm{T}$ cells. Median and ranges are shown ( $\left.\mathrm{n}=17\right)$. B) Percentage of Ki67 ${ }^{+}$cells in HIV-infected $\mathrm{T}_{\mathrm{CD} 32^{\mathrm{dim}}}$ and $\mathrm{T}_{\mathrm{CD} 32^{-}}$ subsets are shown $(n=17)$. Median and ranges are shown. C) Percentage of Ki67 expression on $T_{C D 32}{ }^{\text {dim }}$ cells after immune complexes engagement (Plasma $\mathrm{HIV}^{+}$). Medium alone, FcRs blockers and plasma from an HIV-negative individual were included as controls. Median with interquartile range is shown $(n=8)$. D) Percentage of Ki67 expression on HIV-infected $\mathrm{T}_{\mathrm{CD} 32}$ - cells incubated under the same experimental conditions as shown in $\mathbf{C}$. Median with interquartile range is represented $(n=8)$. Statistical comparisons consisted of the Wilcoxon matched-pairs signed rank test. ${ }^{*} p<0.05 ;{ }^{* *} p<0.01 ;{ }^{* * *} p<0.001 ;{ }^{* * *} p<0.0001$. 


\section{Figure 6}

A

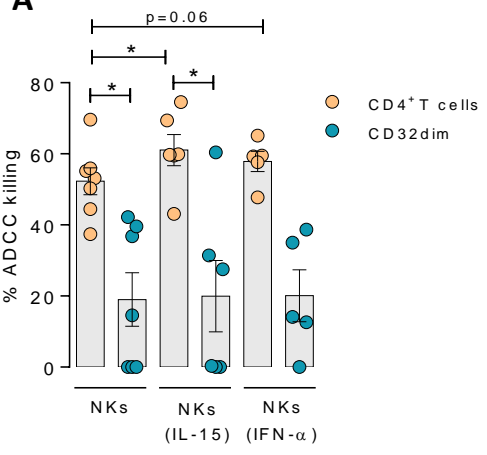

B

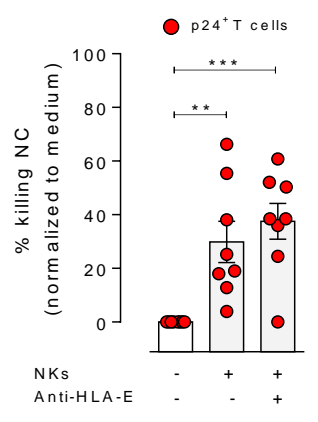

C

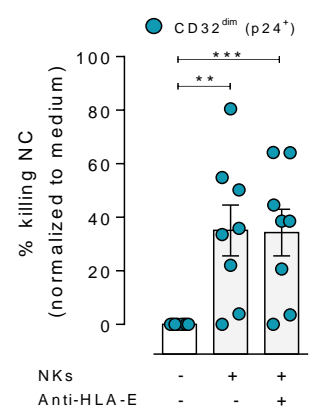

D

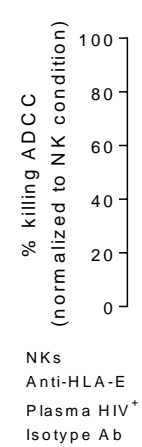

E

O $\mathrm{p}^{24^{+}} \mathrm{T}$ cells

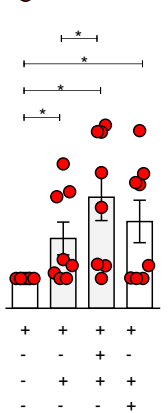

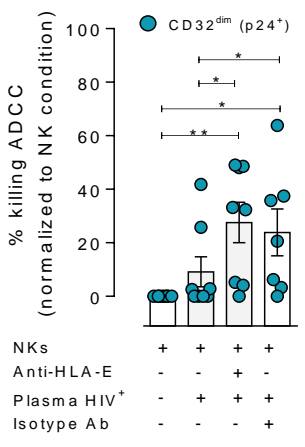

Figure 6. Effect of IL-15, IFN- $\alpha$ and anti-HLA-E antibody in the NK function. CD4 ${ }^{+}$T cells from ART-suppressed participants, either coated with a gp120 recombinant protein (A) or, ex vivo infected with $\operatorname{HIV}_{\mathrm{NL} 4.3}$ (B to E), were subjected to Natural Cytotoxicity (NC) (NK condition) and ADCC (NK + plasma) assays in the presence of cytokines or blocking antibodies. A) Percentage of ADCC killing of HIVgp120-coated CD4 ${ }^{+} \mathrm{T}$ cells by autologous NK cells after treatment with IL-15 or IFN- $\alpha$. Mean with SEM is represented. B-E) Graphs showing the percentage of reduction in HIV-infected cells after using anti-HLA-E antibodies or isotype controls in NC and ADCC functional assays $(n=8)$, in total

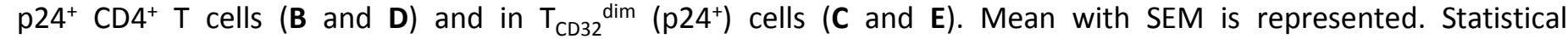
comparisons consisted of the One Sample T test or the Wilcoxon matched-pairs signed rank test. ${ }^{*} p<0.05 ;{ }^{* *} p<0.01$. 
A

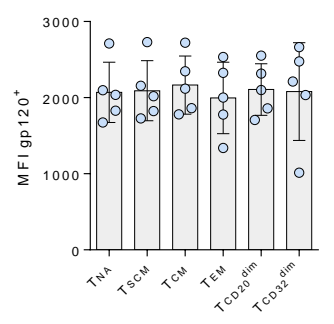

B
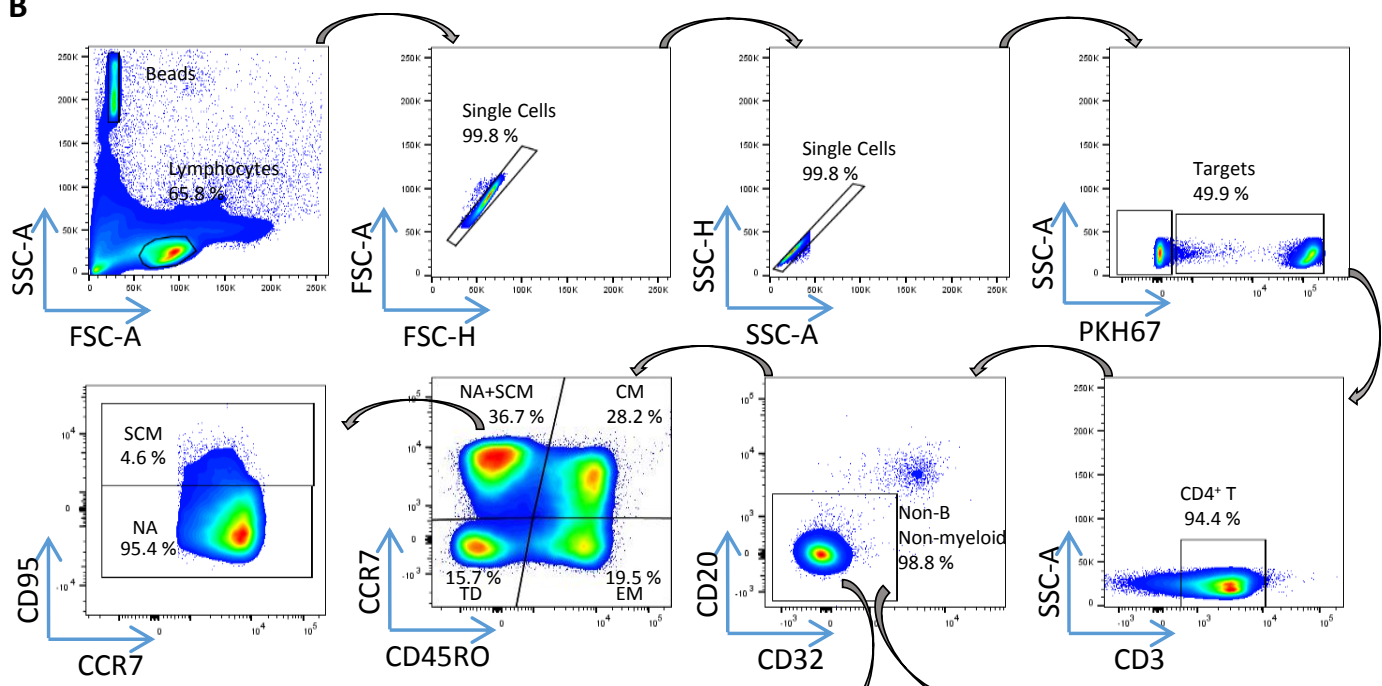

CD45RO

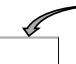

PKH67

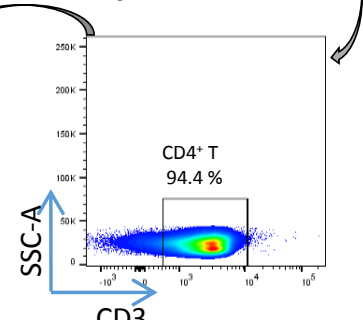

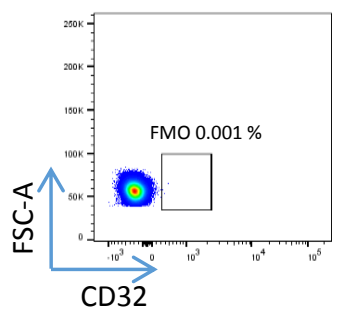

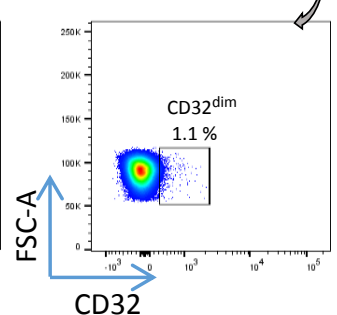

c
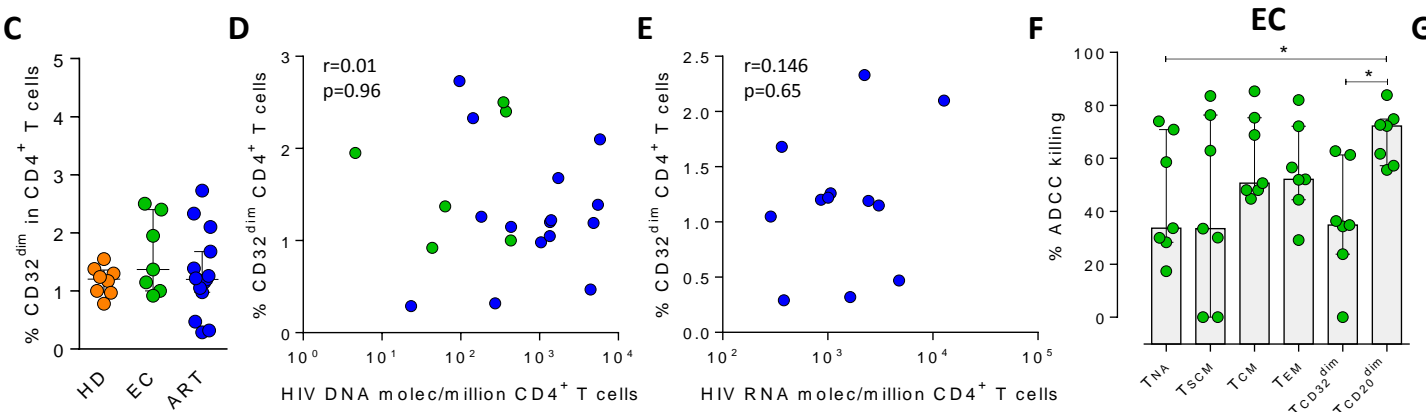

G
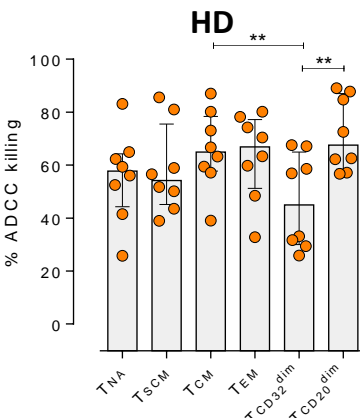

Figure S1. Gp120 cell coating efficiency, gating strategy for the NK-killing assays, and percentages of ADCC killing in elite controllers (EC), and healthy donors (HD). A) Cell coating with recombinant gp120 HIV protein. Detection was performed by flow cytometry using the anti-gp120 antibody A32, and a FITC-labelled anti-human secondary antibody. Mean Fluorescence Intensity (MFI) values are shown. B) Gating strategy used to identify cell killing after the ADCC assay in the different cell subsets. Cell doublets were excluded by forward and side scatter signals and $B$ and myeloid cells discarded based on their high expression of CD20 and CD32. Beads for absolute cell counting were included to calculate ADCC killing by measuring the disappearance of cells in each cell subset. C) Frequency of expression measured by flow cytometry of CD32 on $\mathrm{CD}^{+}{ }^{+} \mathrm{T}$ cells from healthy donors (HD, $\mathrm{n}=8$ ), Elite controllers $(E C, n=7)$ and ART-treated and virologically-suppressed PLWH (ART, $n=15)$. Median with interquartile range are shown. Statistical comparisons were performed using the Mann-Whitney test. $* * * p<0.001 ; * * * * p<0.0001$. D-E) Correlations of the HIV-reservoir size and CD32 expression. Spearman correlations are shown in samples from HIVinfected individuals. ART-treated and EC are shown in blue and green dots, respectively. D) Spearman correlation between the total HIV DNA reservoir size and the frequency of expression of CD32 in CD4 ${ }^{+}$T cells. ART-treated and EC are shown in blue and green dots, respectively. E) Spearman correlation between HIV RNA levels in CD4 ${ }^{+} \mathrm{T}_{\text {cells }}$ and the frequency of expression of CD32 in CD4+ T cells. F-G) Intrinsic susceptibility to NK-mediated ADCC of Naïve $\left(T_{N A}\right)$, Stem Cell Memory $\left(T_{S C M}\right)$, Central Memory $\left(T_{C M}\right)$, Effector Memory $\left(T_{E M}\right), T_{C D 32} \operatorname{dim}$ and $T_{C D 20} \operatorname{dim}$ subsets in $\left.F\right)$ EC, and G) HD. Median with interquartile range is shown. Statistical comparisons were performed using the ANOVA Friedman test. ${ }^{*} p<0.05 ; * * p<0.01$. 
Figure S2

A

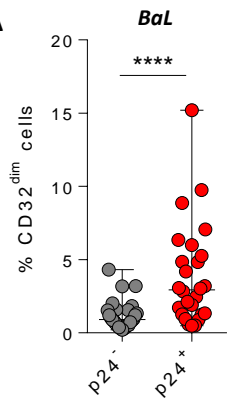

B

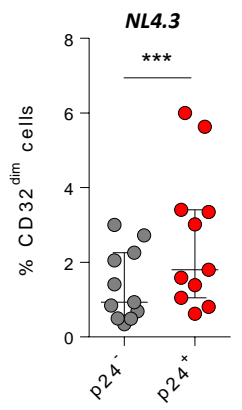

C

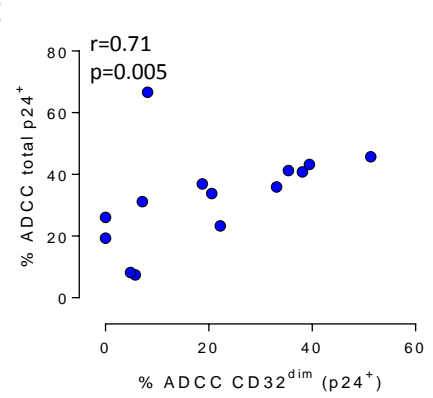

Figure S2. Percentage of CD32 expression on uninfected cells (p24-) and HIV-infected cells (p24+) with BaL ( $n=26)$ in (A) or with NL4.3 ( $n=11)$ in (B) after 5 days of infection. Median with interquartile range are shown. Statistical analyses consisted of the Wilcoxon matched-pairs signed rank test. C) Spearman correlation between the percentage of ADCC-killing of the total HIV-infected $\mathrm{CD}^{+} \mathrm{T}$ cells and the percentage of ADCC-killing of the $\mathrm{T}_{\mathrm{CD} 32}{ }^{\text {dim }}$ subset is shown. 
Figure S4

A
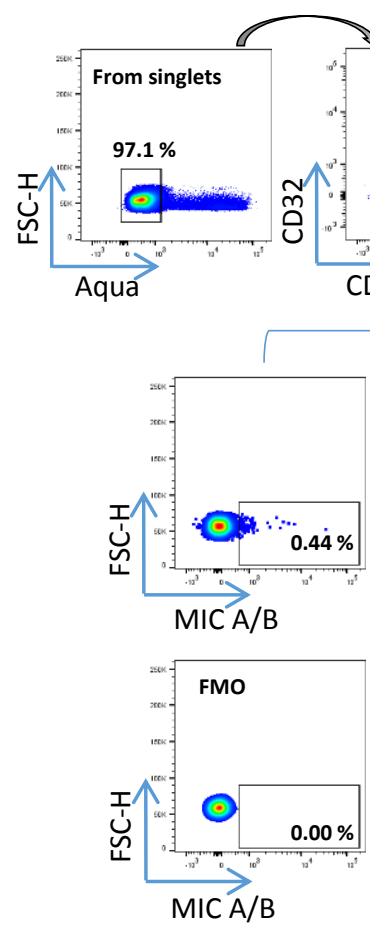
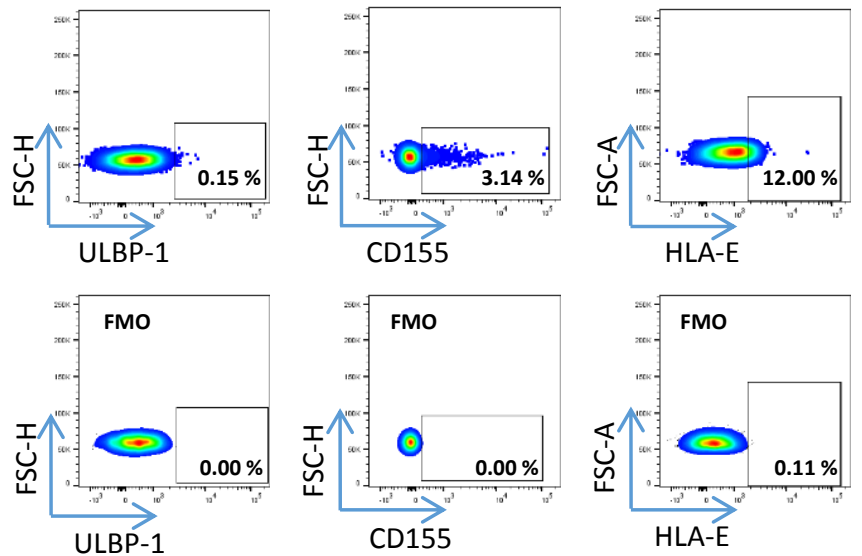

B

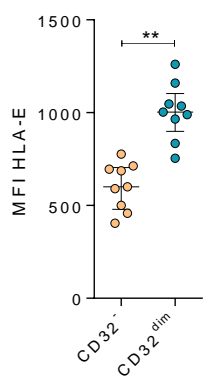

Figure S4. NK-ligands on HIV-infected cells. A) Representative flow cytometry gating strategy used to quantify the expression of MIC A/B, ULBP-1, CD155, and HLA-E ligands on HIV-infected cells after ex vivo infection. FMO controls are also shown. B) Mean Fluorescence Intensity (MFI) values of HLA-E expression on CD4 ${ }^{+}$T cells in the absence of HIV infection. Median with interquartile range is shown. Statistical comparison consisted of the Wilcoxon matched-pairs signed rank test. $* * p<0.01$. 
Figure S5

A

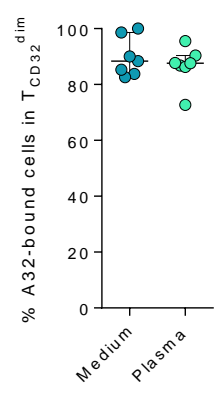

B

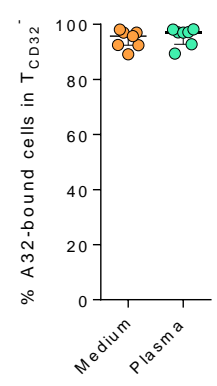

C

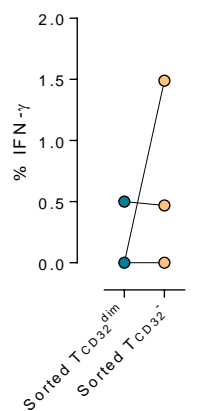

Figure S5. Binding of A32 to T cells and percentage of IFN- $\gamma$ in cell conjugates. The binding capability of the A32 HIV-specific antibody (APC-labeled) to gp120-coated $\mathrm{T}_{\mathrm{CD} 32}{ }^{\text {dim }}$ and $\mathrm{T}_{\mathrm{CD} 32}{ }^{-}$before and after incubation with plasma containing non-HIV specific IgGs, was measured by flow cytometry. A) Percentage of $A 32^{+} T_{C D 32}{ }^{d i m}$ cells. B) Percentage of $\mathrm{A32}^{+} \mathrm{T}_{\mathrm{CD} 32}{ }^{-}$cells. C) Percentage of IFN- $\gamma^{+} \mathrm{NK}$ cells in cell conjugates with sorted $\mathrm{T}_{\mathrm{CD} 32}{ }^{\operatorname{dim}}$ and $\mathrm{T}_{\mathrm{CD} 32}{ }^{-}$ cells after ADCC assays. Statistical comparisons were performed using the Wilcoxon matched-pairs signed rank test. ${ }^{*} p<0.05$. 